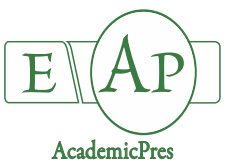

Zhou H et al. (2020)
Notulae Botanicae Horti Agrobotanici Cluj-Napoca 48(4):2263-2278
DOI: $10.15835 /$ nbha48411780
Research Article

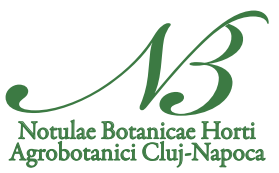

\title{
Spatial and temporal distribution of mung bean (Vigna radiata) and soybean (Glycine $\max$ ) roots
}

\author{
Hang ZHOU ${ }^{2}$, Dianfeng ZHENG ${ }^{1 *}$, Naijie FENG ${ }^{1,3 *}$ \\ ${ }^{1}$ Guangdong Ocean University, College of Agriculture, 1 Haida road, Mazhang district, Zhanjiang, Guangdong, \\ China; byndfnj@126.com (*corresponding author) \\ ${ }^{2}$ Heilongjiang Bayi Agricultural University, College of Agriculture, 5 Xinfeng road, Gaoxin district, Daqing, Heilongiang, \\ China; 18346662760@163.com; byndzdf@126.com ("corresponding author) \\ ${ }^{3}$ Shenzhen Institute of Guangdong Ocean University, 3 Binhaier Road, Dapeng New District, Shenzhen, Guangdong, China
}

\begin{abstract}
Spatial and temporal distribution of roots of mung bean and soybean originated from different geographical backgrounds is an important scientific issue. The aim of this study was to research the spatial and temporal distribution of roots system of soybean cultivar 'Hefeng55' and mung bean cultivar 'Jilv7' which can elucidate differences between soybean roots and mung bean roots in the key spatial and temporal locations. The roots at V6, R2, R4, R5, R6, and R7 stages were collected to acquire data of root length, root surface area, root volume and root dry weight. $49.8 \%, 11.7 \%, 13.2 \%, 14.7 \%$ and $10.6 \%$ of soybean roots and $57.8 \%, 10.7 \%$, $11.2 \%, 11.9 \%$ and $8.4 \%$ of mung bean roots were in $0-5,5-10,10-15,15-20$ and $20-25 \mathrm{~cm}$ horizontal soil layers, respectively; $79.2 \%, 11.5 \%, 4.3 \%, 1.8 \%, 1.1 \%, 1.0 \%$ and $1.1 \%$ of soybean roots and $70.0 \%, 12.3 \%, 8.0 \%, 3.0 \%$, $1.6 \%, 1.7 \%$ and $3.4 \%$ of mung bean roots were in $0-20,20-40,40-60,60-80,80-100,100-120$ and $120-140 \mathrm{~cm}$ vertical soil layers, respectively. Compared with mung bean, soybean had a much larger root system during development. In horizontal direction, soybean root tended to be more laterally developed, but the distribution of mung bean root was more uniform in vertical direction. With a greater root surface area to weight ratio (AWR), mung bean had a finer root system than soybean. These findings can help to clarify the fourdimensional spatial and temporal distribution characteristics of legumes and may provide reference for production practice of soybean and mung bean in the future.
\end{abstract}

Keywords: mung bean; root length; root surface area; root volume; root dry weight; soybean

\section{Introduction}

Roots are an important organ of plants (Fang, 2011) which determines the ability of plants to absorb water and nutrients (Vamerali et al., 2003; Ehdaie et al., 2010). Roots of different crops have different distribution characteristics (Lynch, 1995; Benjamin and Nielsen, 2006). Comparing the temporal and spatial distribution characteristics of different crops' roots is beneficial to research the root structure differences and the adaptability of root systems to the soil environment among different crops (Gan, 2009; Fan et al., 2016).

The distribution characteristic of roots is the basic attribute during plant development (Atta et al., 2013). Pulses tend to have larger root volumes; this may better facilitate moisture absorption and transport to 
other plant parts (Eissenstat and Yanai, 2002; Waisel and Eshel, 2002). Liu et al. (2011) studied the distribution of pulses and found that surface area was mainly distributed in 0-60 cm soil layer. Benjamin and Nielsen (2006) reported $97 \%$ of the root dry weight of soybean and about $80 \%$ of the root dry weight of chickpea and field pea were in the surface $23 \mathrm{~cm}$. Mitchell and Russell (1971) found that $90 \%$ or more of the root dry weight of soybean was concentrated in the upper $7.5 \mathrm{~cm}$ early in the season and in the upper $15 \mathrm{~cm}$ during the remainder of the season.

Both soybean and mung bean are leguminous crops, which originated from different geographical backgrounds. Various workers have studied the root distribution of soybean in the last few years (Calonego et al., 2010; Farmaha et al., 2012), but the difference between these two crops' root distribution at different time and space points is still unclear. In this study, we used innovative horizontal and vertical devices to study the root distribution of soybean and mung bean in 0-5, 5-10, 10-15, 15-20 and 20-25 cm horizontal soil layers and in $0-20,20-40,40-60,60-80,80-100,100-120$ and $120-140 \mathrm{~cm}$ vertical soil layers, respectively. We hypothesized that the root system of soybean and mung bean have different trends in temporal distribution and different pattern in spatial distribution. It can give a deeper understanding of the four-dimensional spatial and temporal distribution characteristics of legume roots and may provide reference for breeding new cultivars of soybean and mung bean in the future.

\section{Materials and Methods}

\section{Experimental site}

The experiment was carried out at outdoor test site in National Coarse Cereals Engineering Research Centre, Daqing, China on June 5, 2015 (soybean) and June 5, 2016 (mung bean). The annual precipitation at the experimental site was $508.7 \mathrm{~mm}$, the average annual temperature was $5.60^{\circ} \mathrm{C}$, the effective accumulated temperature was 2900-3000 ${ }^{\circ} \mathrm{C}$, and the sunshine duration was $1158 \mathrm{~h}$ (Collected from Daqing Weather Station).

\section{Experimental devices}

There were two kinds of devices: horizontal device and vertical device (Figure 1). The horizontal device was a cylindrical metal barrel with a diameter of $50 \mathrm{~cm}$ and a height of $50 \mathrm{~cm}$, which was fixed with a cross steel frame with a length of $54 \mathrm{~cm}$ on both sides of the metal barrel. The inside of the metal barrel was equipped with a diameter of $10 \mathrm{~cm}, 20 \mathrm{~cm}, 30 \mathrm{~cm}$ and $40 \mathrm{~cm}$ of metal net. The distance between the metal net was $5 \mathrm{~cm}$ and the metal net were fixed on the cross-steel frame by nylon straps (Figure 2). The vertical device was a cylindrical plastic barrel with a diameter of $30 \mathrm{~cm}$ and a height of $150 \mathrm{~cm}$ (Figure 3). In order to facilitate sampling, inside the vertical device was a plastic water belt of $30 \mathrm{~cm}$ in diameter and the soil was filled in the plastic water belt (Figure 4). The lower end of the plastic water belt was sealed and four round holes were cut using scissors. 


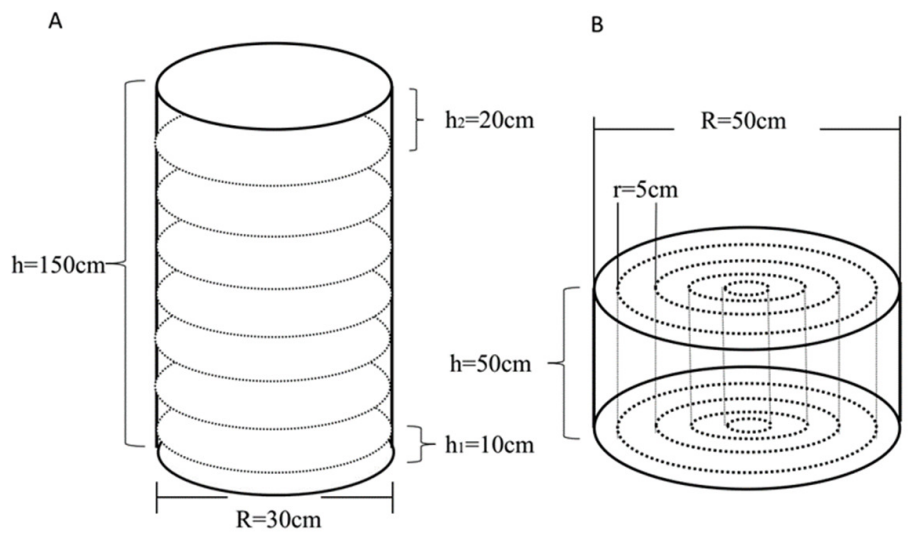

Figure 1. A: Vertical device, which was a cylindrical plastic barrel with a diameter of $30 \mathrm{~cm}$ and a height of $150 \mathrm{~cm}$; B: Horizontal device, which was a cylindrical metal barrel with a diameter of $50 \mathrm{~cm}$ and a height of $50 \mathrm{~cm}$.

Figure 2. Horizontal device entity

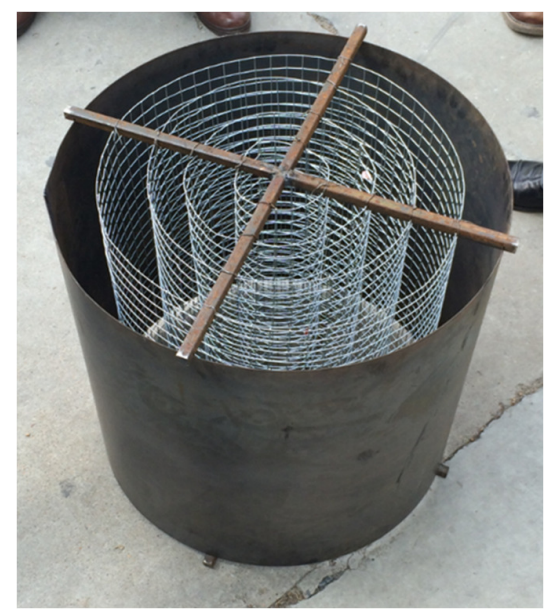

Figure 3. Vertical device entity

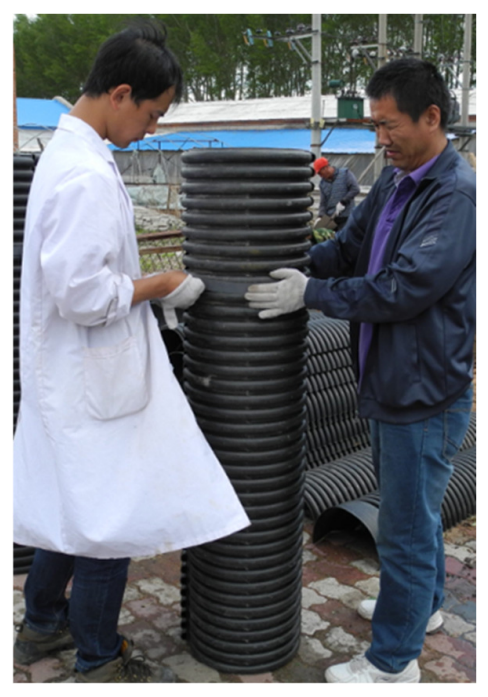




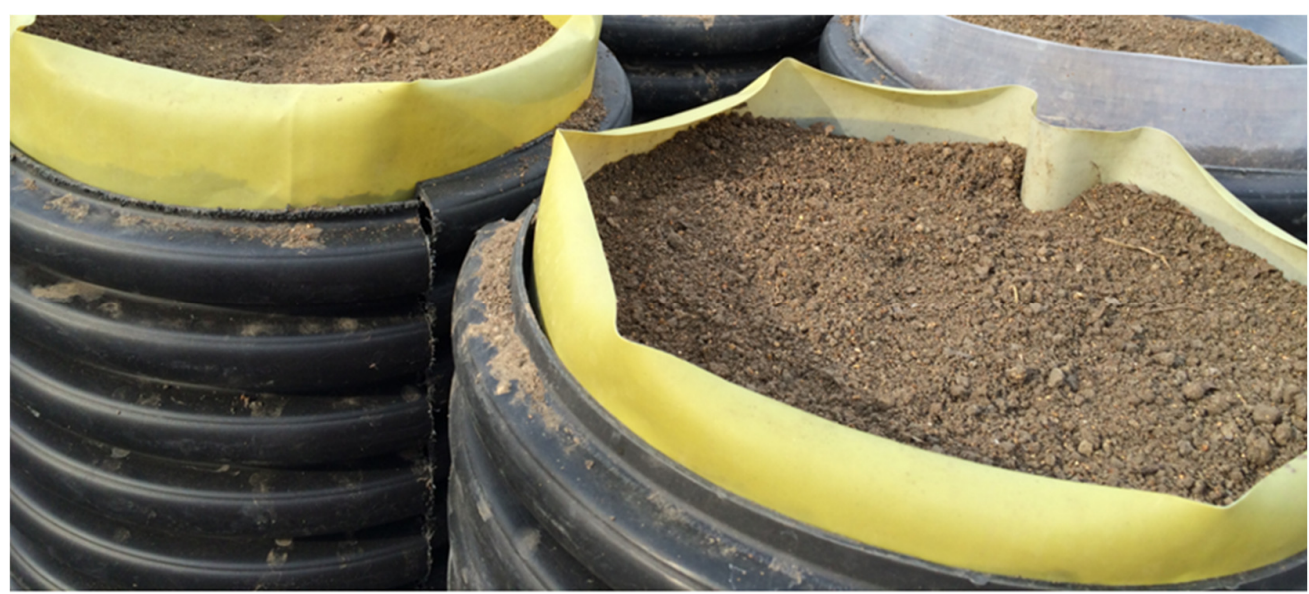

Figure 4. The plastic water belt of $30 \mathrm{~cm}$ in diameter inside the vertical device and the soil was filled in the plastic water belt

\section{Soil characteristics}

The soil was chernozem, with physical and chemical properties characterized by a $\mathrm{pH}$ of 7.8 , effective phosphorus of $13.69 \mathrm{mg} \cdot \mathrm{kg}^{-1}$, alkali-hydrolyzed nitrogen of $134 \mathrm{mg} \cdot \mathrm{kg}^{-1}$, available potassium of $204 \mathrm{mg} \cdot \mathrm{kg}^{-1}$, and organic matter of $32.8 \mathrm{~g} \cdot \mathrm{kg}^{-1}$. The soil was screened before pouring into the devices to remove grass root, tree root and large granular clods and stones. Then the soil was filled into the vertical device and horizontal device, respectively $\left(1.15 \times 102 \mathrm{~kg} \cdot \mathrm{m}^{-3}\right.$ in density).

\section{Experiment design, species and seeding}

Soybean cultivar 'Hefeng55' and mung bean cultivar 'Jilv7' were planted at five seeds per barrel separately in 48 barrels which included 24 horizontal devices and 24 vertical devices. Two seedlings were retained, and grown with four replications per growth stage and type of device.

\section{Root sample collection}

Soil samples were collected from the devices at V6, R2, R4, R5, R6 and R7 stage (Pedersen et al., 2004). In horizontal devices, the centre of the cross section of the column was taken as the starting point to obtain root samples in the horizontal direction i.e. $0-5 \mathrm{~cm}, 5-10 \mathrm{~cm}, 10-15 \mathrm{~cm}, 15-20 \mathrm{~cm}$ and 20-25 cm layers. In vertical devices, the upper soil surface was taken as the starting point to obtain soil samples with root in $0-20$ $\mathrm{cm}, 20-40 \mathrm{~cm}, 40-60 \mathrm{~cm}, 60-80 \mathrm{~cm}, 80-100 \mathrm{~cm}, 100-120 \mathrm{~cm}$ and $120-140 \mathrm{~cm}$ soil layers in vertical direction. The plants were clipped at cotyledons by scissors before sampling. Soil samples containing root were soaked in a plastic bucket filled with water until the soil became soft and then filtered. The obtained root samples were washed with clean tap water and then placed in a plastic, sealable bag, and the bag was placed in a refrigerator for further use.

\section{Data collection}

The harvested root samples were placed in a clear glass tray filled with water. The roots were washed to remove soil particles and other dirt that could hamper efficient scanning of root samples. The glass tray was placed on a scanner (Epson V700) and digital images were generated at 400 dpi. Digital image analysis of root samples was conducted using WinRHIZO (version 2014a, Reagent Instruments Inc., Quebec, Canada) and the data included root length, root surface area and root volume, from which root length density (RLD), root surface area density (RSAD) and root volume density (RVD) were estimated as follows:

$$
\begin{aligned}
& \mathrm{RLD}=\mathrm{L} / \mathrm{V}_{0} \\
& \mathrm{RSAD}=\mathrm{S} / \mathrm{V}_{0} \\
& \mathrm{RVD}=\mathrm{V} / \mathrm{V}_{0}
\end{aligned}
$$


$\mathrm{V}_{0}=\pi \mathrm{r}^{2} \mathrm{~h}$

where $\mathrm{V}$ is the root volume, $\mathrm{L}$ is root length, $\mathrm{S}$ is root surface area, $\mathrm{V}_{0}$ is the soil volume, $\mathrm{r}$ is the radius, and $\mathrm{h}$ is the height.

After scanning, the roots were removed from glass tray and subsequently were placed in an oven at 105 ${ }^{\circ} \mathrm{C}$ for 2 hours, then drying to constant weight in $75^{\circ} \mathrm{C}$ oven. The dry weight of roots was obtained by analytical balance and the root dry weight density (RDWD) was estimated as:

$\mathrm{RDWD}=\mathrm{M} / \mathrm{V}_{0}$

$\mathrm{V}_{0}=\pi \mathrm{r}^{2} \mathrm{~h}$

where $\mathrm{M}$ is the root dry weight.

\section{Statistical analysis}

Differences between soybean and mung bean roots were determined by LSD test by SPSS 22. Figure preparation was carried out by MicroCal Origin software 2017 (Origin Lab).

\section{Results}

\section{Temporal distribution of total root length}

The growth stage needed to reach the maximum total root length for 'Hefeng55' was R5. The greatest total root length of 'Jilv7' were found at R4 in vertical devices and R5 in horizontal devices. In vertical devices, the total root length of 'Jilv7' were greater than 'Hefeng55' at V6 and R2, but 'Hefeng55' had significantly greater total root length than 'Jilv7' by $87.8 \%, 106.6 \%$ and $104.2 \%$ at R5, R6 and R7, respectively. In horizontal devices, 'Hefeng55' had greater total root length than 'Jilv7' from V6 to R2. Total root length of 'Hefeng55' were $59.4 \%$ and $75.1 \%$ significantly more than 'Jilv7' at R2 and R6, respectively (Figure 5).

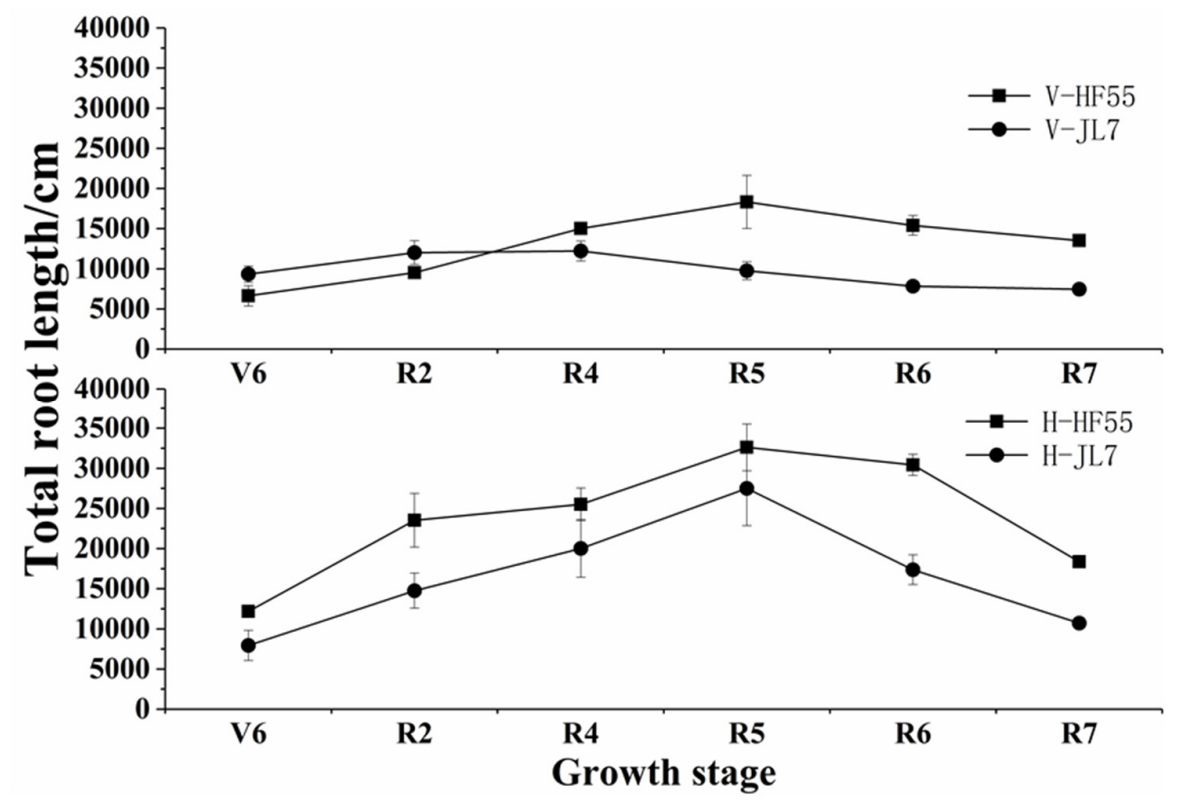

Figure 5. Changes of total root length of soybean and mung bean in vertical device and horizontal device at different growth stages

V-JL7 (mung bean cultivar 'Jilv7' in vertical device), V-HF55 (soybean cultivar 'Hefeng55' in vertical device), H-JL7 (mung bean cultivar 'Jilv7' in horizontal device), H-HF55 (soybean cultivar 'Hefeng55' in horizontal device); Data represent average \pm standard error. Significant at the 0.05 probability level 


\section{The spatial distribution of root length density}

Root length density as estimated by horizontal device: In horizontal devices, the root length density of 'Hefeng55' in different soil layers at R2, R4 and R6 were greater than 'Jilv7', and 'Hefeng55' had significantly greater root length density than 'Jilv7' by $42.5 \%$ in $0-5 \mathrm{~cm}$ soil layer at R6. At V6, R5, and R7, 'Jilv7' had significantly greater root length density than 'Hefeng 55 ' by $57.1 \%, 45.1 \%$ and $32.9 \%$ respectively in $0-5 \mathrm{~cm}$ soil layer. The percentage of root length in 0-5, 5-10, 10-15, 15-20 and 20-25 cm horizontal soil layers to total root length of 'Hefeng 55 ' were $8.8 \%, 15.6 \%, 23.8 \%, 28.9 \%$ and $22.9 \%$, respectively, and the percentage of root length in $0-5,5-10,10-15,15-20$ and $20-25 \mathrm{~cm}$ horizontal soil layers to total root length of 'Jilv7' were $13.6 \%$, $16.8 \%, 21.7 \%, 27.4 \%$ and $20.5 \%$, respectively (Table 1 ).

Root length density as estimated by vertical device: 'Hefeng55' and 'Jilv7' had the largest root length density in $0-20 \mathrm{~cm}$ soil layer in vertical devices. 'Hefeng 55 ' had significantly greater root length density than 'Jilv7' in $0-20 \mathrm{~cm}$ soil layer at R5, R6 and R7 by $88.0 \%, 314.4 \%$ and $141.5 \%$ respectively. The root length density of 'Jilv7' was significantly greater than 'Hefeng 55 ' by $75.9 \%$ in $0-20 \mathrm{~cm}$ soil layer at V6. At R2, 'Jilv7' had significantly greater root length density than 'Hefeng 55 ' in $20-40 \mathrm{~cm}$ soil layer by $119.0 \%$. At R5 and R7, total root length of 'Hefeng55' were $131.2 \%$ and $159.8 \%$ respectively significantly more than 'Jilv7' in $20-40 \mathrm{~cm}$ soil layer. At R6, total root length of 'Jilv7' was 46.1\% significantly more than 'Hefeng55' in 40-60 cm soil layer. The percentage of root length in 0-20, 20-40, 40-60, 60-80, 80-100, 100-120 and 120-140 cm vertical soil layers to total root length of 'Hefeng 55 ' were $56.8 \%, 23.2 \%, 8.6 \%, 4.3 \%, 2.5 \%, 2.1 \%$ and $2.5 \%$, respectively, and the percentage of root length in $0-20,20-40,40-60,60-80,80-100,100-120$ and $120-140 \mathrm{~cm}$ vertical soil layers to total root length of 'Jilv7' were $51.2 \%, 22.9 \%, 13.7 \%, 5.0 \%, 2.2 \%, 2.8 \%$ and $2.2 \%$, respectively (Table 2).

Table 1. Root length density $\left(\mathrm{cm} \cdot \mathrm{cm}^{-3}\right)$ of soybean cultivar 'Hefeng55' and mung bean cultivar 'Jilv7' in different horizontal soil layers at V6, R2, R4, R5, R6 and R7 growth stages

\begin{tabular}{|c|c|c|c|c|c|c|}
\hline \multirow{2}{*}{$\begin{array}{c}\text { Growth } \\
\text { stages }\end{array}$} & \multirow{2}{*}{ Cultivars } & \multicolumn{5}{|c|}{ Soil layers $(\mathrm{cm})$} \\
\cline { 3 - 7 } & & $0-5$ & $5-10$ & $10-15$ & $15-20$ & $20-25$ \\
\hline \multirow{2}{*}{ V6 } & 'Hefeng55' & $0.296 \pm 0.033 \mathrm{a}$ & $0.121 \pm 0.023 \mathrm{a}$ & $0.115 \pm 0.013 \mathrm{a}$ & $0.124 \pm 0.010 \mathrm{a}$ & $0.111 \pm 0.009 \mathrm{a}$ \\
\cline { 2 - 7 } & 'Jilv7' & $0.465 \pm 0.080 \mathrm{~b}$ & $0.104 \pm 0.019 \mathrm{a}$ & $0.078 \pm 0.015 \mathrm{a}$ & $0.072 \pm 0.036 \mathrm{a}$ & $0.039 \pm 0.022 \mathrm{a}$ \\
\hline \multirow{2}{*}{ R2 } & 'Hefeng55' & $0.599 \pm 0.083 \mathrm{a}$ & $0.340 \pm 0.041 \mathrm{a}$ & $0.313 \pm 0.077 \mathrm{a}$ & $0.226 \pm 0.040 \mathrm{a}$ & $0.136 \pm 0.015 \mathrm{a}$ \\
\cline { 2 - 7 } & 'Jilv7' & $0.463 \pm 0.043 \mathrm{a}$ & $0.258 \pm 0.024 \mathrm{a}$ & $0.231 \pm 0.045 \mathrm{a}$ & $0.118 \pm 0.036 \mathrm{a}$ & $0.060 \pm 0.017 \mathrm{a}$ \\
\hline \multirow{2}{*}{ R4 } & 'Hefeng55' & $0.615 \pm 0.115 \mathrm{a}$ & $0.319 \pm 0.036 \mathrm{a}$ & $0.317 \pm 0.039 \mathrm{a}$ & $0.270 \pm 0.014 \mathrm{a}$ & $0.161 \pm 0.013 \mathrm{a}$ \\
\cline { 2 - 7 } & 'Jilv7' & $0.545 \pm 0.142 \mathrm{a}$ & $0.304 \pm 0.092 \mathrm{a}$ & $0.178 \pm 0.026 \mathrm{a}$ & $0.150 \pm 0.037 \mathrm{a}$ & $0.116 \pm 0.024 \mathrm{a}$ \\
\hline \multirow{2}{*}{ R5 } & 'Hefeng55' & $0.643 \pm 0.027 \mathrm{a}$ & $0.513 \pm 0.021 \mathrm{a}$ & $0.441 \pm 0.052 \mathrm{a}$ & $0.312 \pm 0.041 \mathrm{a}$ & $0.193 \pm 0.033 \mathrm{a}$ \\
\cline { 2 - 7 } & 'Jilv7' & $0.933 \pm 0.270 \mathrm{~b}$ & $0.398 \pm 0.089 \mathrm{a}$ & $0.293 \pm 0.033 \mathrm{a}$ & $0.315 \pm 0.092 \mathrm{a}$ & $0.168 \pm 0.023 \mathrm{a}$ \\
\hline \multirow{2}{*}{ R6 } & 'Hefeng55' & $0.724 \pm 0.174 \mathrm{a}$ & $0.356 \pm 0.040 \mathrm{a}$ & $0.350 \pm 0.033 \mathrm{a}$ & $0.353 \pm 0.044 \mathrm{a}$ & $0.193 \pm 0.032 \mathrm{a}$ \\
\cline { 2 - 7 } & 'Jilv7' & $0.508 \pm 0.023 \mathrm{~b}$ & $0.198 \pm 0.031 \mathrm{a}$ & $0.184 \pm 0.028 \mathrm{a}$ & $0.190 \pm 0.021 \mathrm{a}$ & $0.119 \pm 0.020 \mathrm{a}$ \\
\hline \multirow{2}{*}{ R7 } & 'Hefeng55' & $0.334 \pm 0.061 \mathrm{a}$ & $0.230 \pm 0.047 \mathrm{a}$ & $0.195 \pm 0.024 \mathrm{a}$ & $0.214 \pm 0.015 \mathrm{a}$ & $0.132 \pm 0.017 \mathrm{a}$ \\
\cline { 2 - 7 } & 'Jilv7' & $0.444 \pm 0.069 \mathrm{~b}$ & $0.124 \pm 0.013 \mathrm{a}$ & $0.109 \pm 0.009 \mathrm{a}$ & $0.118 \pm 0.012 \mathrm{a}$ & $0.061 \pm 0.005 \mathrm{a}$ \\
\hline
\end{tabular}

Data represent average \pm standard error. Distinct letters in the row indicate significant differences. Significant at the 0.05 probability level 
Table 2. Root length density $\left(\mathrm{cm} \cdot \mathrm{cm}^{-3}\right)$ of soybean cultivar 'Hefeng55' and mung bean cultivar 'Jilv7' in different vertical soil layers at V6, R2, R4, R5, R6 and R7 growth stages

\begin{tabular}{|c|c|c|c|c|c|c|c|c|}
\hline \multirow{2}{*}{$\begin{array}{l}\text { Growth } \\
\text { stages }\end{array}$} & \multirow{2}{*}{ Cultivars } & \multicolumn{7}{|c|}{ Soil layers $(\mathrm{cm})$} \\
\hline & & $0-20$ & $20-40$ & $40-60$ & $60-80$ & $80-100$ & $100-120$ & $120-140$ \\
\hline \multirow{2}{*}{ V6 } & 'Hefeng5' & $\begin{array}{c}0.270 \pm \\
0.074 \mathrm{a}\end{array}$ & $\begin{array}{c}0.098 \pm \\
0.012 \mathrm{a}\end{array}$ & $\begin{array}{c}0.040 \pm \\
0.030 \mathrm{a}\end{array}$ & $\begin{array}{c}0.035 \pm \\
0.012 \mathrm{a}\end{array}$ & $\begin{array}{c}0.016 \pm \\
0.002\end{array}$ & $\begin{array}{c}0.011 \pm \\
0.006\end{array}$ & 0 \\
\hline & 'Jilv7' & $\begin{array}{c}0.475 \pm \\
0.047 b\end{array}$ & $\begin{array}{c}0.115 \pm \\
0.046 \mathrm{a}\end{array}$ & $\begin{array}{c}0.051 \pm \\
0.028 \mathrm{a}\end{array}$ & $\begin{array}{c}0.026 \pm \\
0.014 a\end{array}$ & 0 & 0 & 0 \\
\hline \multirow{2}{*}{$\mathrm{R} 2$} & 'Hefeng55' & $\begin{array}{c}0.361 \pm \\
0.057 \mathrm{a} \\
\end{array}$ & $\begin{array}{c}0.126 \pm \\
0.009 a\end{array}$ & $\begin{array}{c}0.082 \pm \\
0.006 a\end{array}$ & $\begin{array}{c}0.056 \pm \\
0.015 \mathrm{a}\end{array}$ & $\begin{array}{c}0.028 \pm \\
0.006 \mathrm{a}\end{array}$ & $\begin{array}{c}0.015 \pm \\
0.004\end{array}$ & $\begin{array}{c}0.006 \pm \\
0.003\end{array}$ \\
\hline & 'Jilv7' & $\begin{array}{c}0.392 \pm \\
0.081 \mathrm{a}\end{array}$ & $\begin{array}{c}0.276 \pm \\
0.146 b\end{array}$ & $\begin{array}{c}0.154 \pm \\
0.059 \mathrm{a}\end{array}$ & $\begin{array}{c}0.026 \pm \\
0.011 \mathrm{a}\end{array}$ & $\begin{array}{c}0.003 \pm \\
0.001 \mathrm{a}\end{array}$ & 0 & 0 \\
\hline \multirow{2}{*}{ R4 } & 'Hefeng55' & $\begin{array}{c}0.730 \pm \\
0.170 \mathrm{a}\end{array}$ & $\begin{array}{r}0.270 \pm \\
0.068 \mathrm{a}\end{array}$ & $\begin{array}{r}0.116 \pm \\
0.022 \mathrm{a}\end{array}$ & $\begin{array}{r}0.058 \pm \\
0.009 \mathrm{a}\end{array}$ & $\begin{array}{c}0.034 \pm \\
0.006 \mathrm{a}\end{array}$ & $\begin{array}{c}0.031 \pm \\
0.007 \mathrm{a}\end{array}$ & $\begin{array}{c}0.053 \pm \\
0.011\end{array}$ \\
\hline & 'Jilv7' & $\begin{array}{c}0.593 \pm \\
0.132 \mathrm{a}\end{array}$ & $\begin{array}{c}0.167 \pm \\
0.025 a\end{array}$ & $\begin{array}{c}0.085 \pm \\
0.031 \mathrm{a}\end{array}$ & $\begin{array}{c}0.016 \pm \\
0.006 a\end{array}$ & $\begin{array}{c}0.004 \\
\pm 0.002 \mathrm{a}\end{array}$ & $\begin{array}{c}4.68 \times 10^{-4} \pm \\
1.96 \times 10^{-4} \mathrm{a}\end{array}$ & 0 \\
\hline \multirow{2}{*}{ R5 } & 'Hefeng55' & $\begin{array}{c}0.594 \pm \\
0.063 \mathrm{a}\end{array}$ & $\begin{array}{c}0.259 \pm \\
0.047 \mathrm{a}\end{array}$ & $\begin{array}{c}0.093 \pm \\
0.023 \mathrm{a}\end{array}$ & $\begin{array}{r}0.034 \pm \\
0.007 \mathrm{a}\end{array}$ & $\begin{array}{c}0.023 \pm \\
0.002 \mathrm{a}\end{array}$ & $\begin{array}{c}0.033 \pm \\
0.009 \mathrm{a}\end{array}$ & $\begin{array}{c}0.030 \pm \\
0.004 a\end{array}$ \\
\hline & 'Jilv7' & $\begin{array}{c}0.316 \pm \\
0.093 b\end{array}$ & $\begin{array}{c}0.112 \pm \\
0.019 b\end{array}$ & $\begin{array}{c}0.074 \pm \\
0.012 \mathrm{a}\end{array}$ & $\begin{array}{c}0.059 \pm \\
0.006 \mathrm{a}\end{array}$ & $\begin{array}{c}0.049 \pm \\
0.005 \mathrm{a}\end{array}$ & $\begin{array}{c}0.058 \pm \\
0.024 \mathrm{a}\end{array}$ & $\begin{array}{c}0.022 \pm \\
0.012 \mathrm{a}\end{array}$ \\
\hline \multirow{2}{*}{ R6 } & 'Hefeng55' & $\begin{array}{c}0.750 \pm \\
0.080 \mathrm{a}\end{array}$ & $\begin{array}{c}0.226 \pm \\
0.031 \mathrm{a}\end{array}$ & $\begin{array}{c}0.041 \pm \\
0.005 \mathrm{a}\end{array}$ & $\begin{array}{c}0.019 \pm \\
0.002 \mathrm{a}\end{array}$ & $\begin{array}{c}0.011 \pm \\
0.002 \mathrm{a}\end{array}$ & $\begin{array}{c}0.013 \pm \\
0.001 \mathrm{a}\end{array}$ & $\begin{array}{c}0.022 \pm \\
0.005 \mathrm{a}\end{array}$ \\
\hline & 'Jilv7' & $\begin{array}{c}0.181 \pm \\
0.007 \mathrm{~b}\end{array}$ & $\begin{array}{c}0.168 \pm \\
0.040 \mathrm{a}\end{array}$ & $\begin{array}{c}0.130 \pm \\
0.003 \mathrm{~b}\end{array}$ & $\begin{array}{c}0.036 \pm \\
0.010 \mathrm{a}\end{array}$ & $\begin{array}{c}0.0118 \pm \\
0.0002 \mathrm{a}\end{array}$ & $\begin{array}{c}0.026 \pm \\
0.014 \mathrm{a}\end{array}$ & $\begin{array}{c}0.002 \pm \\
7.68 \times 10^{-6} \mathrm{a}\end{array}$ \\
\hline \multirow{2}{*}{ R7 } & 'Hefeng55' & $\begin{array}{c}0.442 \pm \\
0.076 \mathrm{a} \\
\end{array}$ & $\begin{array}{c}0.304 \pm \\
0.044 \mathrm{a}\end{array}$ & $\begin{array}{c}0.106 \pm \\
0.026 \mathrm{a}\end{array}$ & $\begin{array}{r}0.037 \pm \\
0.007 \mathrm{a} \\
\end{array}$ & $\begin{array}{c}0.026 \pm \\
0.008 \mathrm{a}\end{array}$ & $\begin{array}{l}0.016 \pm \\
0.004 \mathrm{a}\end{array}$ & $\begin{array}{c}0.026 \pm \\
0.010 \mathrm{a} \\
\end{array}$ \\
\hline & 'Jilv7' & $\begin{array}{l}0.183 \pm \\
0.031 b\end{array}$ & $\begin{array}{c}0.117 \pm \\
0.016 \mathrm{~b}\end{array}$ & $\begin{array}{r}0.080 \pm \\
0.011 \mathrm{a}\end{array}$ & $\begin{array}{r}0.044 \pm \\
0.012 \mathrm{a}\end{array}$ & $\begin{array}{c}0.023 \pm \\
0.010 \mathrm{a}\end{array}$ & $\begin{array}{c}0.034 \pm \\
0.025 \mathrm{a}\end{array}$ & $\begin{array}{c}0.069 \pm \\
0.037 \mathrm{a}\end{array}$ \\
\hline
\end{tabular}

Data represent average \pm standard error. Distinct letters in the row indicate significant differences. Significant at the 0.05 probability level.

\section{Temporal distribution of total root surface area}

The greatest total root surface area of 'Hefeng55' was found at R5. The greatest total root surface of 'Jilv7' were found at R2 in vertical devices and R5 in horizontal devices. In vertical devices, the total root surface of 'Hefeng55' were greater than 'Jilv7' except for V6, and 'Hefeng55' were 132.5\%, 226.1\%, 225.7\% and $247.7 \%$ respectively significantly more than 'Jilv7' at R4, R5, R6 and R7. In horizontal devices, the total root surface of 'Hefeng55' were greater than 'Jilv7' during all growth stages. In addition to V6, 'Hefeng55' had significantly greater total root surface than 'Jilv7' by $93.9 \%, 104.7 \%, 90.6 \%, 145.5 \%$ and $141.8 \%$, respectively (Figure 6).

\section{The spatial distribution of root surface area density}

Root surface area density as estimated by horizontal device: The greatest root surface area density of 'Hefeng 55' and 'Jilv7' were found in 0-5 cm soil layers in horizontal devices. During all growth stages, the root surface area density of 'Hefeng 55 ' in different soil layers were greater than 'Jilv7' except for the $0-5 \mathrm{~cm}$ soil layer at V6. 'Hefeng55' had significantly greater root surface area density than 'Jilv7' by $64.3 \%, 55.7 \%$, and $81.1 \%$ respectively at R2, R4, and R6 in $0-5 \mathrm{~cm}$ soil layer. In addition, the root surface area density of 'Hefeng 55 ' were respectively significantly greater than 'Jilv7' by $68.8 \%, 114.9 \%, 156.9 \%$, and 157.6 in $5-10 \mathrm{~cm}$ soil layer at R2, R5, R6, and R7. Significant differences also were in $10-15 \mathrm{~cm}$ soil layer at R4, R5, R6 and R7 and root surface area density of 'Hefeng 55 ' were $144.5 \%, 150.4 \%, 173.2 \%$, and $161.8 \%$ respectively more than 'Jilv7'. In 15-20 cm soil layer at R6 and R7, 'Hefeng55' had significantly greater root surface area density than 'Jilv7' by $162.8 \%$ and $168.4 \%$ respectively. The percentage of root surface area in $0-5,5-10,10-15,15-20$ and $20-25 \mathrm{~cm}$ horizontal soil layers to total root surface area of 'Hefeng 55 ' were $10.6 \%, 16.0 \%, 23.6 \%, 28.0 \%$ and $21.8 \%$, 
respectively, and the percentage of root surface area in 0-5, 5-10, 10-15, 15-20 and 20-25 cm horizontal soil layers to total root surface area of 'Jilv7' were $15.8 \%, 16.7 \%, 21.2 \%, 26.3 \%$ and $20.0 \%$, respectively (Table 3 ).

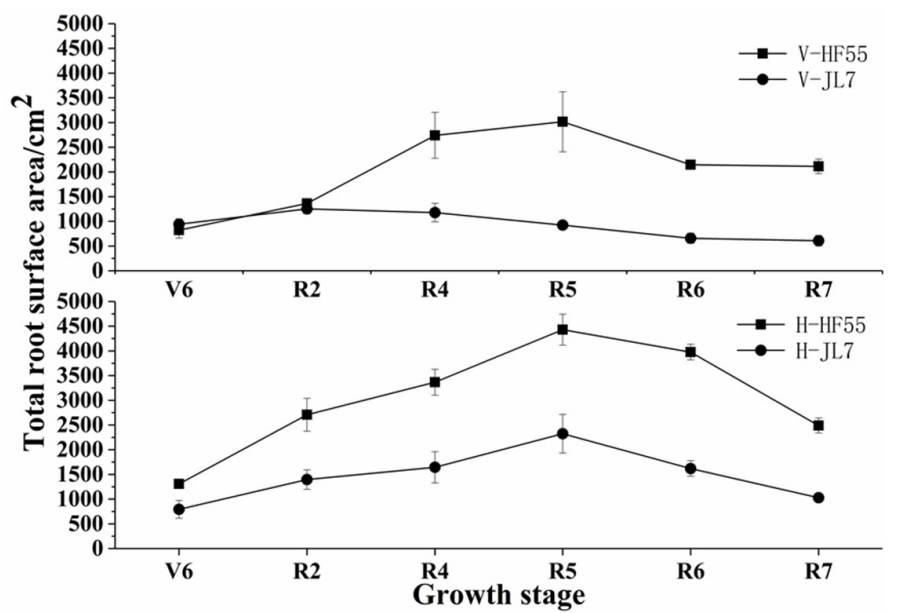

Figure 6. Changes of total root surface area of soybean and mung bean in vertical device and horizontal device at different growth stages

V-JL7 (mung bean cultivar 'Jilv7' in vertical device), V-HF55 (soybean cultivar 'Hefeng55' in vertical device), H-JL7 (mung bean cultivar 'Jilv7' in horizontal device), H-HF55 (soybean cultivar 'Hefeng55' in horizontal device); Data represent average \pm standard error. Significant at the 0.05 probability level

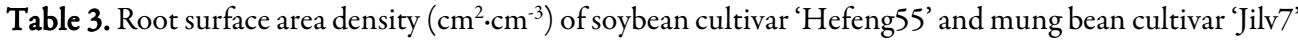
in different horizontal soil layers at V6, R2, R4, R5, R6 and R7 growth stages

\begin{tabular}{|c|c|c|c|c|c|c|}
\hline \multirow{2}{*}{$\begin{array}{c}\text { Growth } \\
\text { stages }\end{array}$} & \multirow{2}{*}{ Cultivars } & \multicolumn{5}{|c|}{ Soil layers $(\mathrm{cm})$} \\
\cline { 3 - 6 } & & $0-5$ & $5-10$ & $10-15$ & $15-20$ & $20-25$ \\
\hline \multirow{2}{*}{ V6 } & 'Hefeng55' & $42.24 \pm 4.82 \mathrm{a}$ & $13.41 \pm 1.86 \mathrm{a}$ & $12.72 \pm 1.11 \mathrm{a}$ & $12.49 \pm 0.70 \mathrm{a}$ & $11.10 \pm 1.01 \mathrm{a}$ \\
\cline { 2 - 7 } & 'Jilv7' & $49.98 \pm 7.63 \mathrm{a}$ & $9.64 \pm 1.71 \mathrm{a}$ & $7.57 \pm 1.35 \mathrm{a}$ & $7.16 \pm 3.51 \mathrm{a}$ & $3.93 \pm 2.11 \mathrm{a}$ \\
\hline \multirow{2}{*}{ R2 } & 'Hefeng55' & $84.55 \pm 9.51 \mathrm{a}$ & $39.93 \pm 4.63 \mathrm{a}$ & $34.91 \pm 7.69 \mathrm{a}$ & $24.62 \pm 3.82 \mathrm{a}$ & $15.42 \pm 1.80 \mathrm{a}$ \\
\cline { 2 - 7 } & 'Jilv7' & $51.47 \pm 3.83 \mathrm{~b}$ & $23.65 \pm 1.52 \mathrm{~b}$ & $21.28 \pm 4.09 \mathrm{a}$ & $10.96 \pm 3.45 \mathrm{a}$ & $5.58 \pm 1.47 \mathrm{a}$ \\
\hline \multirow{2}{*}{ R4 } & 'Hefeng55' & $94.06 \pm 13.73 \mathrm{a}$ & $42.57 \pm 4.40 \mathrm{a}$ & $40.57 \pm 4.61 \mathrm{a}$ & $35.20 \pm 2.48 \mathrm{a}$ & $20.75 \pm 1.89 \mathrm{a}$ \\
\cline { 2 - 7 } & 'Jilv7' & $60.41 \pm 12.41 \mathrm{~b}$ & $28.15 \pm 7.98 \mathrm{a}$ & $16.59 \pm 2.29 \mathrm{~b}$ & $13.68 \pm 3.28 \mathrm{a}$ & $10.61 \pm 2.10 \mathrm{a}$ \\
\hline \multirow{2}{*}{ R5 } & 'Hefeng55' & $105.41 \pm 4.48 \mathrm{a}$ & $71.27 \pm 3.95 \mathrm{a}$ & $59.70 \pm 6.24 \mathrm{a}$ & $41.79 \pm 4.68 \mathrm{a}$ & $24.28 \pm 3.99 \mathrm{a}$ \\
\cline { 2 - 7 } & 'Jilv7' & $85.97 \pm 3.12 \mathrm{a}$ & $33.16 \pm 7.47 \mathrm{~b}$ & $23.84 \pm 2.75 \mathrm{~b}$ & $26.26 \pm 7.16 \mathrm{a}$ & $14.29 \pm 2.01 \mathrm{a}$ \\
\hline \multirow{2}{*}{ R6 } & 'Hefeng55' & $107.87 \pm 19.43 \mathrm{a}$ & $48.99 \pm 4.99 \mathrm{a}$ & $45.80 \pm 3.97 \mathrm{a}$ & $44.20 \pm 4.86 \mathrm{a}$ & $24.44 \pm 4.53 \mathrm{a}$ \\
\cline { 2 - 7 } & 'Jilv7' & $59.57 \pm 3.35 \mathrm{~b}$ & $19.07 \pm 2.78 \mathrm{~b}$ & $16.76 \pm 2.41 \mathrm{~b}$ & $16.82 \pm 1.90 \mathrm{~b}$ & $10.49 \pm 1.71 \mathrm{a}$ \\
\hline \multirow{2}{*}{ R7 } & 'Hefeng55' & $58.62 \pm 9.24 \mathrm{a}$ & $32.77 \pm 7.20 \mathrm{a}$ & $26.28 \pm 3.74 \mathrm{a}$ & $27.78 \pm 1.35 \mathrm{a}$ & $16.87 \pm 1.37 \mathrm{a}$ \\
\cline { 2 - 7 } & 'Jilv7' & $54.13 \pm 7.43 \mathrm{a}$ & $12.72 \pm 0.93 \mathrm{~b}$ & $10.04 \pm 0.59 \mathrm{~b}$ & $10.35 \pm 0.84 \mathrm{~b}$ & $5.27 \pm 0.36 \mathrm{a}$ \\
\hline
\end{tabular}

Data represent average \pm standard error. Distinct letters in the row indicate significant differences. Significant at the 0.05 probability level

Root surface area density as estimated by vertical device: The greatest root surface density of 'Hefeng 55' and 'Jilv7' were found in 0-20 cm soil layer. At R4, R5, R6, and R7, the root surface density of 'Hefeng55' were respectively significantly greater than 'Jilv7' by $126.1 \%, 217.3 \%, 273.3 \%$ and $456.7 \%$ in $0-20 \mathrm{~cm}$ soil layer. In $20-40 \mathrm{~cm}$ soil layer, the root surface density of 'Hefeng 55 ' were $167.7 \%, 275.8 \%, 345.5 \%$ and $222.6 \%$ respectively significantly more than 'Jilv7' at R4, R5, R6 and R7. The percentage of root surface area in 0-20, $20-40,40-60,60-80,80-100,100-120$ and $120-140 \mathrm{~cm}$ vertical soil layers to total root surface area of 'Hefeng 55 ' were $53.9 \%, 22.6 \%, 8.3 \%, 8.6 \%, 2.3 \%, 2.0 \%$ and $2.3 \%$, respectively, and the percentage of root surface area in $0-20,20-40,40-60,60-80,80-100,100-120$ and $120-140 \mathrm{~cm}$ vertical soil layers to total root surface area of 'Jilv7' were 55.7\%, 19.3\%, 13.7\%, 4.8\%, 2.1\%, 2.7\% and 1.7\%, respectively (Table 4). 
Table 4. Root surface area density $\left(\mathrm{cm}^{2} \cdot \mathrm{cm}^{-3}\right)$ of soybean cultivar 'Hefeng55' and mung bean cultivar' Jilv7' in different vertical soil layers at V6, R2, R4, R5, R6 and R7 growth stages

\begin{tabular}{|c|c|c|c|c|c|c|c|c|}
\hline \multirow{2}{*}{$\begin{array}{c}\text { Growth } \\
\text { stages }\end{array}$} & \multirow{2}{*}{ Cultivars } & \multicolumn{7}{|c|}{ Soil layers $(\mathrm{cm})$} \\
\hline & & $0-20$ & $20-40$ & $40-60$ & $60-80$ & $80-100$ & $100-120$ & $120-140$ \\
\hline \multirow{2}{*}{ V6 } & 'Hefeng55' & $\begin{array}{c}41.81 \pm \\
1.88 \mathrm{a}\end{array}$ & $\begin{array}{c}12.65 \pm \\
2.03 \mathrm{a}\end{array}$ & $\begin{array}{c}5.09 \pm \\
0.35 \mathrm{a}\end{array}$ & $\begin{array}{c}4.38 \pm \\
1.35 \mathrm{a}\end{array}$ & $\begin{array}{c}1.98 \pm \\
0.26\end{array}$ & $\begin{array}{c}0.72 \pm \\
0.14\end{array}$ & 0 \\
\hline & 'Jilv7' & $\begin{array}{c}47.79 \pm \\
4.36 \mathrm{a}\end{array}$ & $\begin{array}{c}11.53 \pm \\
4.55 \mathrm{a}\end{array}$ & $\begin{array}{c}5.29 \pm \\
2.63 \mathrm{a}\end{array}$ & $\begin{array}{c}2.13 \pm \\
1.39 \mathrm{a}\end{array}$ & 0 & 0 & 0 \\
\hline \multirow{2}{*}{ R2 } & 'Hefeng55' & $\begin{array}{c}52.98 \pm \\
6.83 a\end{array}$ & $\begin{array}{c}16.81 \pm \\
0.84 \mathrm{a}\end{array}$ & $\begin{array}{c}11.97 \pm \\
1.24 \mathrm{a}\end{array}$ & $\begin{array}{c}7.70 \pm \\
1.86 \mathrm{a}\end{array}$ & $\begin{array}{c}3.91 \pm \\
0.82 \mathrm{a}\end{array}$ & $\begin{array}{c}2.22 \pm \\
0.60\end{array}$ & $\begin{array}{c}1.25 \pm \\
0.44\end{array}$ \\
\hline & 'Jilv7' & $\begin{array}{r}60.33 \pm \\
11.79 \mathrm{a}\end{array}$ & $\begin{array}{c}17.23 \pm \\
2.47 \mathrm{a}\end{array}$ & $\begin{array}{r}9.07 \pm \\
3.29 \mathrm{a}\end{array}$ & $\begin{array}{c}1.78 \pm \\
0.62 \mathrm{a}\end{array}$ & $\begin{array}{c}0.36 \pm \\
0.18 \mathrm{a}\end{array}$ & 0 & 0 \\
\hline \multirow{2}{*}{$\mathrm{R} 4$} & 'Hefeng55' & $\begin{array}{c}107.13 \pm \\
21.61 \mathrm{a}\end{array}$ & $\begin{array}{r}43.56 \pm \\
10.14 \mathrm{a}\end{array}$ & $\begin{array}{c}17.70 \pm \\
2.85 \mathrm{a}\end{array}$ & $\begin{array}{c}8.48 \pm \\
0.99 \mathrm{a}\end{array}$ & $\begin{array}{c}4.82 \pm \\
0.62 \mathrm{a}\end{array}$ & $\begin{array}{c}4.74 \pm \\
0.79 \mathrm{a}\end{array}$ & $\begin{array}{c}7.48 \pm \\
1.31\end{array}$ \\
\hline & 'Jilv7' & $\begin{array}{c}47.39 \pm \\
5.16 b\end{array}$ & $\begin{array}{c}16.27 \pm \\
3.97 \mathrm{~b}\end{array}$ & $\begin{array}{c}16.52 \pm \\
5.42 \mathrm{a}\end{array}$ & $\begin{array}{c}2.98 \pm \\
1.04 \mathrm{a}\end{array}$ & $\begin{array}{c}0.24 \pm \\
0.20 \mathrm{a}\end{array}$ & $\begin{array}{c}0.06 \pm \\
0.03 \mathrm{a}\end{array}$ & 0 \\
\hline \multirow{2}{*}{ R5 } & 'Hefeng55' & $\begin{array}{c}96.10 \pm \\
12.24 \mathrm{a}\end{array}$ & $\begin{array}{c}43.89 \pm \\
7.64 \mathrm{a}\end{array}$ & $\begin{array}{c}15.20 \pm \\
3.89 \mathrm{a}\end{array}$ & $\begin{array}{c}45.27 \pm \\
9.86 \mathrm{a}\end{array}$ & $\begin{array}{c}3.39 \pm \\
0.26 \mathrm{a}\end{array}$ & $\begin{array}{r}5.00 \pm \\
1.23 \mathrm{a}\end{array}$ & $\begin{array}{c}4.61 \pm \\
0.71 \mathrm{a}\end{array}$ \\
\hline & 'Jilv7' & $\begin{array}{c}30.29 \pm \\
7.61 b\end{array}$ & $\begin{array}{c}11.68 \pm \\
3.47 \mathrm{~b}\end{array}$ & $\begin{array}{c}9.00 \pm \\
1.61 \mathrm{a}\end{array}$ & $\begin{array}{c}5.65 \pm \\
0.33 \mathrm{a}\end{array}$ & $\begin{array}{c}4.73 \pm \\
0.40 \mathrm{a}\end{array}$ & $\begin{array}{l}5.67 \pm \\
2.36 \mathrm{a}\end{array}$ & $\begin{array}{c}2.26 \pm \\
1.08 \mathrm{a}\end{array}$ \\
\hline \multirow{2}{*}{ R6 } & 'Hefeng55' & $\begin{array}{c}70.86 \pm \\
10.75 a\end{array}$ & $\begin{array}{c}48.51 \pm \\
4.97 \mathrm{a}\end{array}$ & $\begin{array}{c}16.40 \pm \\
4.23 \mathrm{a}\end{array}$ & $\begin{array}{c}5.82 \pm \\
0.99 \mathrm{a}\end{array}$ & $\begin{array}{c}3.85 \pm \\
1.02 \mathrm{a}\end{array}$ & $\begin{array}{c}2.42 \pm \\
0.58 \mathrm{a}\end{array}$ & $\begin{array}{c}4.15 \pm \\
1.51 \mathrm{a} \\
\end{array}$ \\
\hline & 'Jilv7' & $\begin{array}{c}18.98 \pm \\
2.68 \mathrm{~b}\end{array}$ & $\begin{array}{c}10.89 \pm \\
1.70 \mathrm{~b}\end{array}$ & $\begin{array}{c}7.76 \pm \\
1.19 \mathrm{a}\end{array}$ & $\begin{array}{c}4.52 \pm \\
1.08 \mathrm{a}\end{array}$ & $\begin{array}{c}2.49 \pm \\
0.96 \mathrm{a}\end{array}$ & $\begin{array}{c}3.50 \pm \\
2.40 \mathrm{a}\end{array}$ & $\begin{array}{c}4.21 \pm \\
3.97 \mathrm{a}\end{array}$ \\
\hline \multirow{2}{*}{ R7 } & 'Hefeng55' & $\begin{array}{c}100.88 \pm \\
9.25 \mathrm{a}\end{array}$ & $\begin{array}{c}31.55 \pm \\
4.31 \mathrm{a}\end{array}$ & $\begin{array}{c}6.33 \pm \\
0.77 \mathrm{a}\end{array}$ & $\begin{array}{c}2.96 \pm \\
0.40 \mathrm{a}\end{array}$ & $\begin{array}{c}1.90 \pm \\
0.28 \mathrm{a}\end{array}$ & $\begin{array}{c}2.02 \pm \\
0.14 \mathrm{a}\end{array}$ & $\begin{array}{c}2.96 \pm \\
0.67 \mathrm{a}\end{array}$ \\
\hline & 'Jilv7' & $\begin{array}{c}18.12 \pm \\
1.48 \mathrm{~b}\end{array}$ & $\begin{array}{l}9.78 \pm \\
1.55 b\end{array}$ & $\begin{array}{c}7.09 \pm \\
0.97 \mathrm{a}\end{array}$ & $\begin{array}{l}2.13 \pm \\
1.18 \mathrm{a}\end{array}$ & $\begin{array}{c}0.77 \pm \\
0.38 \mathrm{a}\end{array}$ & $\begin{array}{c}1.50 \pm \\
1.44 \mathrm{a}\end{array}$ & $\begin{array}{c}0.16 \pm \\
0.08 \mathrm{a}\end{array}$ \\
\hline
\end{tabular}

Data represent average \pm standard error. Distinct letters in the row indicate significant differences. Significant at the 0.05 probability level.

\section{Temporal distribution of total root volume}

During all growth stages, 'Hefeng55' had greater total root volume than 'Jilv7'. In vertical devices, 'Hefeng 55 ' had significantly greater total root volume than 'Jilv7' by $224.4 \%, 472.8 \%, 444.4 \%$ and $374.1 \%$ at R4, R5, R6, and R7, respectively. In horizontal devices, 'Hefeng55' had significantly greater total root volume than 'Jilv7' by $151.0 \%, 184.1 \%, 215.3 \%, 224.1 \%$ and $200.6 \%$ at R2, R4, R5, R6 and R7, respectively. The greatest total root volume of Hengfeng 55 was found at R5, and the greatest total root volume of 'Jilv7' was at $\mathrm{R} 4$ in vertical devices and at $\mathrm{R} 5$ in horizontal devices (Figure 7).

\section{The spatial distribution of root volume density}

Root volume density as estimated by horizontal device: During all growth stages, 'Hefeng 55 ' had greater root volume density than 'Jilv7' in different soil layer in horizontal devices. 'Hefeng55' had significantly greater root volume density than 'Jilv7' in $0-5 \mathrm{~cm}$ soil layer at V6 and $5-10 \mathrm{~cm}$ soil layer at R7 by $37.2 \%$ and $236.4 \%$, respectively. In addition, at R2, R4, R5, and R6, the root volume density of 'Hefeng55' were significantly greater than that of 'Jilv7' in 0-5, 5-10, 10-15 and 15-20 cm soil layer. The percentage of root volume in 0-5, 5-10, 10$15,15-20$ and $20-25 \mathrm{~cm}$ horizontal soil layers to total root volume of 'Hefeng 55 ' were $15.0 \%, 16.0 \%, 22.4 \%$, $26.6 \%$ and $20.0 \%$, respectively, and the percentage of root volume in $0-5,5-10,10-15,15-20$ and $20-25 \mathrm{~cm}$ horizontal soil layers to total root volume of 'Jilv7' were $22.4 \%, 15.9 \%, 19.6 \%, 23.9 \%$ and $18.2 \%$, respectively (Table 5). 


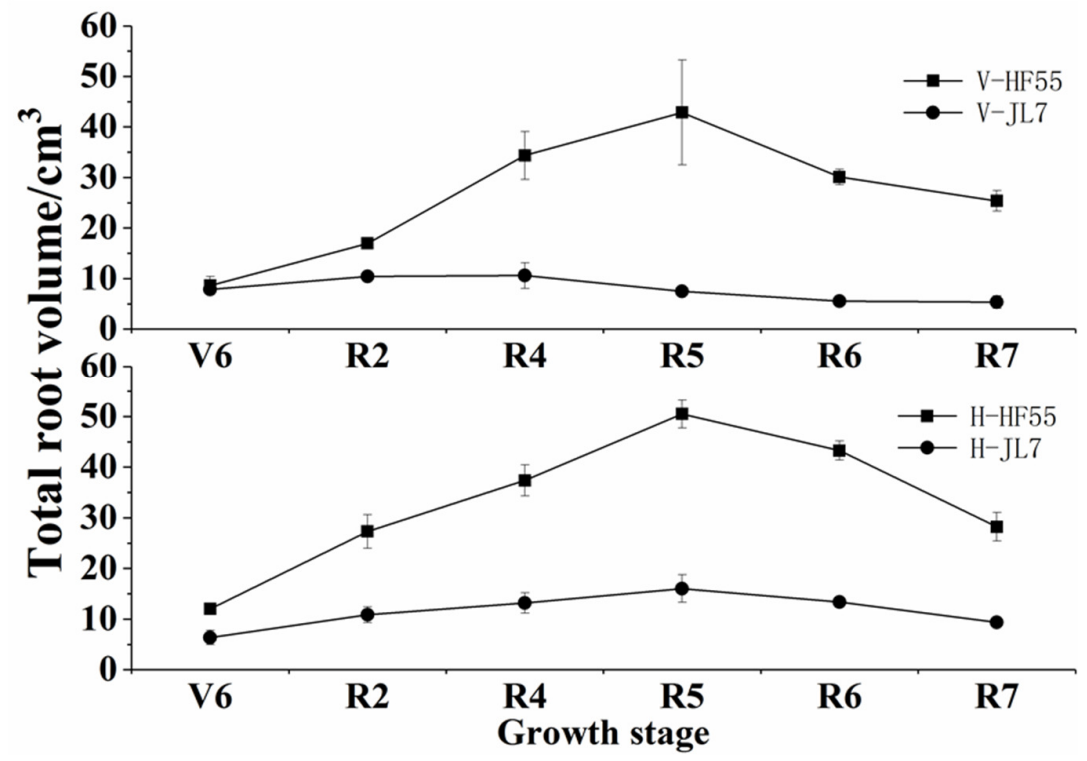

Figure 7. Changes of total root volume of soybean and mung bean in vertical device and horizontal device at different growth stages

V-JL7 (mung bean cultivar 'Jilv7' in vertical device), V-HF55 (soybean cultivar 'Hefeng55' in vertical device), H-JL7 (mung bean cultivar 'Jilv7' in horizontal device), H-HF55 (soybean cultivar 'Hefeng55' in horizontal device); Data represent average \pm standard error. Significant at the 0.05 probability level

Table 5. Root volume density $\left(\mathrm{cm}^{3} \cdot \mathrm{cm}^{-3}\right)$ of soybean cultivar 'Hefeng55' and mung bean cultivar 'Jilv7' in different horizontal soil layers at V 6, R2, R4, R5, R6 and R7

\begin{tabular}{|c|c|c|c|c|c|c|}
\hline \multirow{2}{*}{$\begin{array}{c}\text { Growth } \\
\text { stages }\end{array}$} & \multirow{2}{*}{ Cultivars } & \multicolumn{5}{|c|}{ Soil layers $(\mathrm{cm})$} \\
\cline { 3 - 7 } & & $0-5$ & $5-10$ & $10-15$ & $15-20$ & $20-25$ \\
\hline \multirow{2}{*}{ V6 } & 'Hefeng55' & $0.598 \pm 0.073 \mathrm{a}$ & $0.121 \pm 0.011 \mathrm{a}$ & $0.114 \pm 0.008 \mathrm{a}$ & $0.102 \pm 0.006 \mathrm{a}$ & $0.090 \pm 0.010 \mathrm{a}$ \\
\cline { 2 - 7 } & 'Jilv7' & $0.436 \pm 0.057 \mathrm{~b}$ & $0.071 \pm 0.013 \mathrm{a}$ & $0.059 \pm 0.010 \mathrm{a}$ & $0.057 \pm 0.027 \mathrm{a}$ & $0.031 \pm 0.016 \mathrm{a}$ \\
\hline \multirow{2}{*}{ R2 } & 'Hefeng55' & $1.145 \pm 0.098 \mathrm{a}$ & $0.379 \pm 0.043 \mathrm{a}$ & $0.313 \pm 0.061 \mathrm{a}$ & $0.264 \pm 0.060 \mathrm{a}$ & $0.140 \pm 0.017 \mathrm{a}$ \\
\cline { 2 - 7 } & 'Jilv7' & $0.511 \pm 0.010 \mathrm{~b}$ & $0.175 \pm 0.014 \mathrm{~b}$ & $0.158 \pm 0.032 \mathrm{~b}$ & $0.082 \pm 0.027 \mathrm{~b}$ & $0.042 \pm 0.010 \mathrm{a}$ \\
\hline \multirow{2}{*}{ R4 } & 'Hefeng55' & $1.509 \pm 0.132 \mathrm{a}$ & $0.463 \pm 0.046 \mathrm{a}$ & $0.418 \pm 0.049 \mathrm{a}$ & $0.369 \pm 0.035 \mathrm{a}$ & $0.217 \pm 0.024 \mathrm{a}$ \\
\cline { 2 - 7 } & 'Jilv7' & $0.704 \pm 0.083 \mathrm{~b}$ & $0.210 \pm 0.054 \mathrm{~b}$ & $0.125 \pm 0.016 \mathrm{~b}$ & $0.100 \pm 0.023 \mathrm{~b}$ & $0.078 \pm 0.015 \mathrm{a}$ \\
\hline \multirow{2}{*}{ R5 } & 'Hefeng55' & $1.756 \pm 0.090 \mathrm{a}$ & $0.805 \pm 0.068 \mathrm{a}$ & $0.652 \pm 0.063 \mathrm{a}$ & $0.454 \pm 0.043 \mathrm{a}$ & $0.250 \pm 0.040 \mathrm{a}$ \\
\cline { 2 - 7 } & 'Jilv7' & $0.694 \pm 0.179 \mathrm{~b}$ & $0.222 \pm 0.050 \mathrm{~b}$ & $0.156 \pm 0.018 \mathrm{~b}$ & $0.176 \pm 0.045 \mathrm{~b}$ & $0.097 \pm 0.014 \mathrm{a}$ \\
\hline \multirow{2}{*}{ R6 } & 'Hefeng55' & $1.610 \pm 0.145 \mathrm{a}$ & $0.544 \pm 0.050 \mathrm{a}$ & $0.483 \pm 0.039 \mathrm{a}$ & $0.446 \pm 0.043 \mathrm{a}$ & $0.249 \pm 0.051 \mathrm{a}$ \\
\cline { 2 - 7 } & 'Jilv7' & $0.819 \pm 0.075 \mathrm{~b}$ & $0.153 \pm 0.021 \mathrm{~b}$ & $0.124 \pm 0.017 \mathrm{~b}$ & $0.120 \pm 0.014 \mathrm{~b}$ & $0.074 \pm 0.012 \mathrm{a}$ \\
\hline \multirow{2}{*}{ R7 } & 'Hefeng55' & $0.994 \pm 0.166 \mathrm{a}$ & $0.378 \pm 0.090 \mathrm{a}$ & $0.285 \pm 0.046 \mathrm{a}$ & $0.293 \pm 0.018 \mathrm{a}$ & $0.176 \pm 0.013 \mathrm{a}$ \\
\cline { 2 - 7 } & 'Jilv7' & $0.818 \pm 0.109 \mathrm{a}$ & $0.112 \pm 0.008 \mathrm{~b}$ & $0.077 \pm 0.003 \mathrm{a}$ & $0.074 \pm 0.006 \mathrm{a}$ & $0.037 \pm 0.004 \mathrm{a}$ \\
\hline
\end{tabular}

Data represent average \pm standard error. Distinct letters in the row indicate significant differences. Significant at the 0.05 probability level

Root volume density as estimated by vertical device: In vertical devices, the root volume density of 'Hefeng55' was greater than that of 'Jilv7' in different soil layers during all growth stages except for the $0-20 \mathrm{~cm}$ soil layer at V6. They differed significantly in $0-20 \mathrm{~cm}$ soil layers at R2, R4, R5, R6 and R7, and in $20-40 \mathrm{~cm}$ soil layers at R4, R5, R6, and R7. The root volume density in $0-20 \mathrm{~cm}$ soil layers at R2, R4, R5, R6 and R7 of 'Hefeng 55 ' were $41.6 \%, 194.6 \%, 429.2 \%, 528.3 \%$ and $564.5 \%$ significantly more than these of 'Jilv7', respectively. In 20-40 cm soil layers at R4, R5, R6, and R7, 'Hefeng55' had significantly greater root volume density than 'Jilv7' by $362.2 \%, 752.2 \%, 523.2 \%$ and $340.3 \%$. The greatest proportion of root volume density of 'Hefeng 55' and 'Jilv7' was found in 0-20 cm soil layer. The percentage of root volume in 0-20, 20-40, 40-60, 60-80, 80-100, 100-120 and 120-140 cm vertical soil layers to total root volume of 'Hefeng55' were $57.4 \%$, $23.5 \%, 8.6 \%, 3.9 \%, 2.2 \%, 2.1 \%$ and $2.3 \%$, respectively, and the percentage of root volume in $0-20,20-40,40$ - 
$60,60-80,80-100,100-120$ and $120-140 \mathrm{~cm}$ vertical soil layers to total root volume of 'Jilv7' were $57.8 \%$, $18.6 \%, 12.9 \%, 4.6 \%, 2.1 \%, 2.5 \%$ and $1.5 \%$, respectively (Table 6).

Table 6. Root volume density $\left(\mathrm{cm}^{3} \cdot \mathrm{cm}^{-3}\right)$ of soybean cultivar 'Hefeng55' and mung bean cultivar 'Jilv7' in different vertical soil layers at V6, R2, R4, R5, R6 and R7 growth stages

\begin{tabular}{|c|c|c|c|c|c|c|c|c|}
\hline \multirow{2}{*}{ Growth stages } & \multirow{2}{*}{ Cultivars } & \multicolumn{7}{|c|}{ Soil layers $(\mathrm{cm})$} \\
\hline & & $0-20$ & $20-40$ & $40-60$ & $60-80$ & $80-100$ & $100-120$ & $120-140$ \\
\hline \multirow{2}{*}{ V6 } & 'Hefeng55' & $\begin{array}{l}0.344 \pm \\
0.102 \mathrm{a} \\
\end{array}$ & $\begin{array}{l}0.132 \pm \\
0.026 \mathrm{a} \\
\end{array}$ & $\begin{array}{l}0.054 \pm \\
0.004 \mathrm{a} \\
\end{array}$ & $\begin{array}{l}0.044 \pm \\
0.013 \mathrm{a} \\
\end{array}$ & $\begin{array}{c}0.020 \pm \\
0.002\end{array}$ & $\begin{array}{c}0.016 \pm \\
0.008\end{array}$ & 0 \\
\hline & 'Jilv7’ & $\begin{array}{l}0.399 \pm \\
0.038 \mathrm{a}\end{array}$ & $\begin{array}{l}0.094 \pm \\
0.037 \mathrm{a}\end{array}$ & $\begin{array}{l}0.045 \pm \\
0.020 \mathrm{a}\end{array}$ & $\begin{array}{l}0.018 \pm \\
0.011 \mathrm{a}\end{array}$ & 0 & 0 & 0 \\
\hline \multirow{2}{*}{$\mathrm{R} 2$} & 'Hefeng55' & $\begin{array}{l}0.703 \pm \\
0.064 \mathrm{a} \\
\end{array}$ & $\begin{array}{l}0.184 \pm \\
0.006 \mathrm{a}\end{array}$ & $\begin{array}{r}0.144 \pm \\
0.021 \mathrm{a} \\
\end{array}$ & $\begin{array}{l}0.087 \pm \\
0.018 \mathrm{a} \\
\end{array}$ & $\begin{array}{l}0.044 \pm \\
0.009 \mathrm{a}\end{array}$ & $\begin{array}{c}0.026 \pm \\
0.007\end{array}$ & $\begin{array}{c}0.015 \pm \\
0.006\end{array}$ \\
\hline & 'Jilv7' & $\begin{array}{l}0.497 \pm \\
0.080 \mathrm{~b}\end{array}$ & $\begin{array}{l}0.142 \pm \\
0.020 \mathrm{a}\end{array}$ & $\begin{array}{r}0.077 \pm \\
0.028 \mathrm{a}\end{array}$ & $\begin{array}{l}0.016 \pm \\
0.006 \mathrm{a}\end{array}$ & $\begin{array}{l}0.006 \pm \\
0.001 \mathrm{a}\end{array}$ & 0 & 0 \\
\hline \multirow{2}{*}{$\mathrm{R} 4$} & 'Hefeng55' & $\begin{array}{l}1.382 \pm \\
0.232 \mathrm{a}\end{array}$ & $\begin{array}{l}0.598 \pm \\
0.098 \mathrm{a}\end{array}$ & $\begin{array}{l}0.205 \pm \\
0.051 \mathrm{a}\end{array}$ & $\begin{array}{l}0.070 \pm \\
0.016 \mathrm{a}\end{array}$ & $\begin{array}{l}0.041 \pm \\
0.003 \mathrm{a}\end{array}$ & $\begin{array}{c}0.063 \pm \\
0.014 a\end{array}$ & $\begin{array}{c}0.056 \pm \\
0.010\end{array}$ \\
\hline & 'Jilv7' & $\begin{array}{l}0.454 \pm \\
0.116 \mathrm{~b} \\
\end{array}$ & $\begin{array}{l}0.123 \pm \\
0.027 b\end{array}$ & $\begin{array}{r}0.142 \pm \\
0.039 \mathrm{a} \\
\end{array}$ & $\begin{array}{l}0.028 \pm \\
0.008 \mathrm{a} \\
\end{array}$ & $\begin{array}{l}0.010 \pm \\
0.002 \mathrm{a} \\
\end{array}$ & $\begin{array}{c}0.005 \pm \\
0.001 \mathrm{a}\end{array}$ & 0 \\
\hline \multirow{2}{*}{$\mathrm{R} 5$} & 'Hefeng55' & $\begin{array}{l}1.338 \pm \\
0.215 \mathrm{a}\end{array}$ & $\begin{array}{l}0.568 \pm \\
0.125 \mathrm{a}\end{array}$ & $\begin{array}{r}0.222 \pm \\
0.028 \mathrm{a}\end{array}$ & $\begin{array}{l}0.102 \pm \\
0.009 a\end{array}$ & $\begin{array}{c}0.057 \pm \\
0.006 \mathrm{a}\end{array}$ & $\begin{array}{c}0.059 \pm \\
0.006 \mathrm{a}\end{array}$ & $\begin{array}{c}0.085 \pm \\
0.012 \mathrm{a}\end{array}$ \\
\hline & 'Jilv7' & $\begin{array}{l}0.261 \pm \\
0.043 b\end{array}$ & $\begin{array}{l}0.070 \pm \\
0.012 b\end{array}$ & $\begin{array}{c}0.055 \pm \\
0.006 a\end{array}$ & $\begin{array}{l}0.044 \pm \\
0.001 \mathrm{a}\end{array}$ & $\begin{array}{l}0.036 \pm \\
0.003 \mathrm{a}\end{array}$ & $\begin{array}{l}0.044 \pm \\
0.019 \mathrm{a}\end{array}$ & $\begin{array}{l}0.019 \pm \\
0.008 \mathrm{a}\end{array}$ \\
\hline \multirow{2}{*}{ R6 } & 'Hefeng55' & $\begin{array}{l}1.081 \pm \\
0.124 \mathrm{a}\end{array}$ & $\begin{array}{l}0.635 \pm \\
0.067 \mathrm{a}\end{array}$ & $\begin{array}{r}0.207 \pm \\
0.057 \mathrm{a}\end{array}$ & $\begin{array}{l}0.075 \pm \\
0.013 \mathrm{a} \\
\end{array}$ & $\begin{array}{l}0.048 \pm \\
0.011 \mathrm{a}\end{array}$ & $\begin{array}{c}0.030 \pm \\
0.007 \mathrm{a}\end{array}$ & $\begin{array}{l}0.052 \pm \\
0.020 \mathrm{a}\end{array}$ \\
\hline & 'Jilv7' & $\begin{array}{l}0.172 \pm \\
0.025 b\end{array}$ & $\begin{array}{l}0.121 \pm \\
0.035 b\end{array}$ & $\begin{array}{r}0.061 \pm \\
0.010 \mathrm{a}\end{array}$ & $\begin{array}{l}0.037 \pm \\
0.008 \mathrm{a} \\
\end{array}$ & $\begin{array}{l}0.021 \pm \\
0.007 \mathrm{a}\end{array}$ & $\begin{array}{c}0.029 \pm \\
0.018 \mathrm{a}\end{array}$ & $\begin{array}{c}0.031 \pm \\
0.019 a\end{array}$ \\
\hline \multirow{2}{*}{ R7 } & 'Hefeng55' & $\begin{array}{l}1.220 \pm \\
0.119 \mathrm{a} \\
\end{array}$ & $\begin{array}{l}0.358 \pm \\
0.048 \mathrm{a} \\
\end{array}$ & $\begin{array}{r}0.080 \pm \\
0.010 \mathrm{a} \\
\end{array}$ & $\begin{array}{l}0.038 \pm \\
0.006 \mathrm{a} \\
\end{array}$ & $\begin{array}{l}0.026 \pm \\
0.003 \mathrm{a} \\
\end{array}$ & $\begin{array}{c}0.026 \pm \\
0.002 \mathrm{a} \\
\end{array}$ & $\begin{array}{c}0.033 \pm \\
0.007 \mathrm{a}\end{array}$ \\
\hline & 'Jilv7' & $\begin{array}{l}0.184 \pm \\
0.014 b\end{array}$ & $\begin{array}{l}0.081 \pm \\
0.014 \mathrm{~b}\end{array}$ & $\begin{array}{l}0.060 \pm \\
0.009 \mathrm{a}\end{array}$ & $\begin{array}{l}0.014 \pm \\
0.008 \mathrm{a}\end{array}$ & $\begin{array}{l}0.006 \pm \\
0.003 \mathrm{a}\end{array}$ & $\begin{array}{c}0.010 \pm \\
0.006 \mathrm{a}\end{array}$ & $\begin{array}{r}0.007 \pm \\
0.001 \mathrm{a}\end{array}$ \\
\hline
\end{tabular}

Data represent average \pm standard error. Distinct letters in the row indicate significant differences. Significant at the 0.05 probability level.

\section{Temporal distribution of total root dry weight}

The distribution of total root dry weight showed that 'Hefeng55' had significantly greater root dry weight than 'Jilv7' during all growth stages in horizontal devices, and total root dry weight of 'Hefeng55' were significantly greater than 'Jilv7' except for V6 in vertical devices (Figure 8).

\section{The spatial distribution of root dry weight density}

Root dry weight density as estimated by horizontal device: During all growth stages, the root dry weight density of 'Hefeng55' were significantly greater than 'Jilv7' in different layers in horizontal devices. The percentage of root dry weight in $0-5,5-10,10-15,15-20$ and 20-25 cm horizontal soil layers to total root dry weight of 'Hefeng 55 ' were $49.8 \%, 11.7 \%, 13.2 \%, 14.7 \%$ and $10.6 \%$, respectively, and the percentage of root dry weight in $0-5,5-10,10-15,15-20$ and $20-25 \mathrm{~cm}$ horizontal soil layers to total root dry weight of 'Jilv7' were $57.8 \%, 10.7 \%, 11.2 \%, 11.9 \%$ and $8.4 \%$, respectively (Table 7 ).

Root dry weight density as estimated by vertical device: 'Hefeng55' had significantly greater root dry weight density than 'Jilv7' in 0-20 cm soil layer during all growth stages, in 20-40 cm soil layer at R2, R4, R5, R6, R7 and in 40-60 cm soil layer at R6. The percentage of root dry weight in 0-20, 20-40, 40-60, 60-80, 80$100,100-120$ and 120-140 cm vertical soil layers to total root dry weight of 'Hefeng 55 ' were $79.2 \%, 11.5 \%$, $4.3 \%, 1.8 \%, 1.1 \%, 1.0 \%$ and $1.1 \%$, respectively, and the percentage of root dry weight in 0-20, 20-40, 40-60, 60$80,80-100,100-120$ and $120-140 \mathrm{~cm}$ vertical soil layers to total root dry weight of 'Jilv7' were $70.0 \%, 12.3 \%$, $8.0 \%, 3.0 \%, 1.6 \%, 1.7 \%$ and $3.4 \%$, respectively (Table 8 ). 


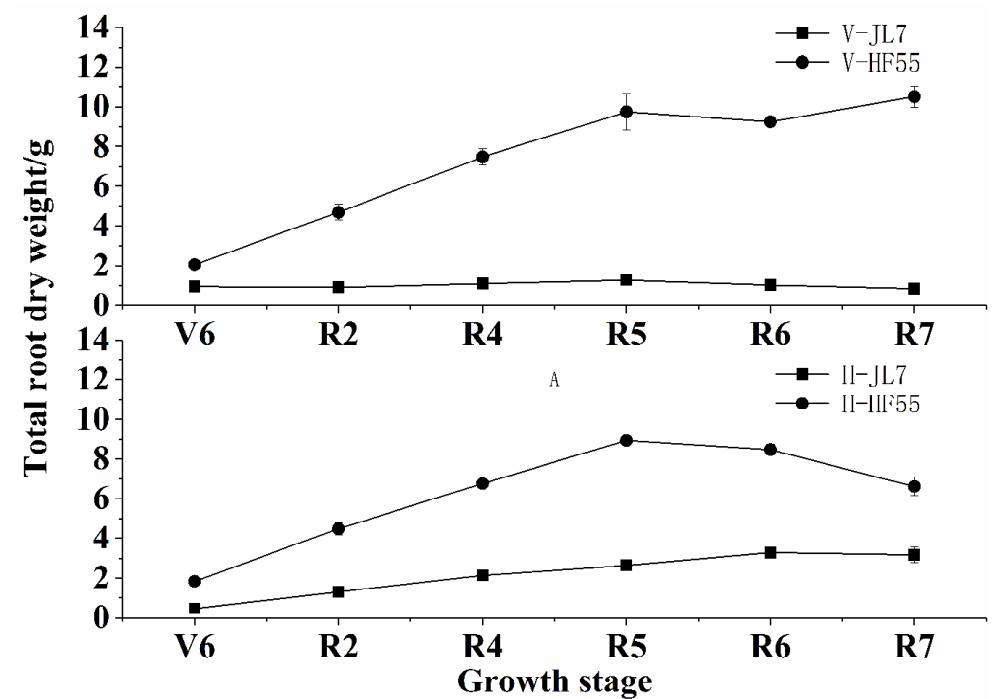

Figure 8. Changes of total root dry weight of soybean and mung bean in vertical device and horizontal device at different growth stages

V-JL7 (mung bean cultivar 'Jilv7' in vertical device), V-HF55 (soybean cultivar 'Hefeng55' in vertical device), H-JL7 (mung bean cultivar 'Jilv7' in horizontal device), H-HF55 (soybean cultivar 'Hefeng55' in horizontal device); Data represent average \pm standard error. Significant at the 0.05 probability level

Table 7. Root dry weight density $\left(\mathrm{g} \cdot \mathrm{m}^{-3}\right)$ of soybean cultivar 'Hefeng 55 ' and mung bean cultivar 'Jilv7' in different horizontal soil layers at V6, R2, R4, R5, R6 and R7 growth stages

\begin{tabular}{|c|c|c|c|c|c|c|}
\hline $\begin{array}{c}\text { Growth } \\
\text { stages }\end{array}$ & \multirow{2}{*}{ Cultivars } & \multicolumn{5}{|c|}{ Soil layers (cm) } \\
\cline { 3 - 7 } & & $0-5$ & $5-10$ & $10-15$ & $15-20$ & $20-25$ \\
\hline \multirow{2}{*}{ V6 } & 'Hefeng55' & $245.06 \pm 27.99 \mathrm{a}$ & $15.19 \pm 1.17 \mathrm{a}$ & $10.85 \pm 0.61 \mathrm{a}$ & $9.53 \pm 0.74 \mathrm{a}$ & $6.32 \pm 0.34 \mathrm{a}$ \\
\cline { 2 - 6 } & 'Jilv7' & $77.67 \pm 10.99 \mathrm{~b}$ & $5.76 \pm 1.46 \mathrm{~b}$ & $2.53 \pm 0.59 \mathrm{~b}$ & $1.33 \pm 0.17 \mathrm{~b}$ & $0.50 \pm 0.10 \mathrm{~b}$ \\
\hline \multirow{2}{*}{ R2 } & 'Hefeng55' & $636.82 \pm 31.53 \mathrm{a}$ & $43.24 \pm 0.67 \mathrm{a}$ & $28.99 \pm 4.14 \mathrm{a}$ & $18.67 \pm 1.90 \mathrm{a}$ & $11.43 \pm 1.29 \mathrm{a}$ \\
\cline { 2 - 7 } & 'Jilv7' & $159.09 \pm 5.48 \mathrm{~b}$ & $12.74 \pm 1.40 \mathrm{~b}$ & $11.70 \pm 1.81 \mathrm{~b}$ & $6.28 \pm 1.00 \mathrm{~b}$ & $3.42 \pm 0.28 \mathrm{~b}$ \\
\hline \multirow{2}{*}{ R4 } & 'Hefeng55' & $813.25 \pm 26.93 \mathrm{a}$ & $70.03 \pm 5.98 \mathrm{a}$ & $48.46 \pm 4.46 \mathrm{a}$ & $38.59 \pm 1.02 \mathrm{a}$ & $20.87 \pm 1.16 \mathrm{a}$ \\
\cline { 2 - 7 } & 'Jilv7' & $309.31 \pm 2.91 \mathrm{~b}$ & $19.67 \pm 1.67 \mathrm{~b}$ & $11.99 \pm 1.33 \mathrm{~b}$ & $8.65 \pm 1.68 \mathrm{~b}$ & $6.22 \pm 1.08 \mathrm{~b}$ \\
\hline \multirow{2}{*}{ R5 } & 'Hefeng55' & $1087.96 \pm 59.58 \mathrm{a}$ & $99.99 \pm 5.56 \mathrm{a}$ & $63.88 \pm 3.53 \mathrm{a}$ & $47.39 \pm 4.54 \mathrm{a}$ & $26.22 \pm 4.24 \mathrm{a}$ \\
\cline { 2 - 7 } & 'Jilv7' & $359.25 \pm 32.01 \mathrm{~b}$ & $25.87 \pm 3.39 \mathrm{~b}$ & $14.89 \pm 0.76 \mathrm{~b}$ & $13.23 \pm 2.01 \mathrm{~b}$ & $7.80 \pm 0.91 \mathrm{~b}$ \\
\hline \multirow{2}{*}{ R6 } & 'Hefeng55' & $1064.84 \pm 75.73 \mathrm{a}$ & $77.76 \pm 7.77 \mathrm{a}$ & $56.00 \pm 5.31 \mathrm{a}$ & $48.32 \pm 2.87 \mathrm{a}$ & $26.71 \pm 4.00 \mathrm{a}$ \\
\cline { 2 - 7 } & 'Jilv7' & $506.16 \pm 30.21 \mathrm{~b}$ & $26.26 \pm 4.22 \mathrm{~b}$ & $17.56 \pm 1.77 \mathrm{~b}$ & $13.84 \pm 2.02 \mathrm{~b}$ & $7.41 \pm 1.56 \mathrm{~b}$ \\
\hline \multirow{2}{*}{ R7 } & 'Hefeng55' & $856.87 \pm 75.52 \mathrm{a}$ & $63.65 \pm 7.81 \mathrm{a}$ & $41.51 \pm 4.94 \mathrm{a}$ & $35.42 \pm 2.83 \mathrm{a}$ & $20.28 \pm 1.08 \mathrm{a}$ \\
\cline { 2 - 7 } & 'Jilv7' & $504.63 \pm 91.54 \mathrm{~b}$ & $28.13 \pm 2.72 \mathrm{~b}$ & $15.63 \pm 2.04 \mathrm{~b}$ & $12.99 \pm 1.82 \mathrm{~b}$ & $5.46 \pm 0.64 \mathrm{~b}$ \\
\hline
\end{tabular}

Data represent average \pm standard error. Distinct letters in the row indicate significant differences. Significant at the 0.05 probability level. 
Table 8. Root dry weight density $\left(\mathrm{g} \cdot \mathrm{m}^{-3}\right)$ of soybean cultivar 'Hefeng55' and mung bean cultivar 'Jilv7' in different vertical soil layers at V6, R2, R4, R5, R6 and R7 growth stages

\begin{tabular}{|c|c|c|c|c|c|c|c|c|}
\hline \multirow{2}{*}{ Growth stages } & \multirow{2}{*}{ Cultivars } & \multicolumn{7}{|c|}{ Soil layers $(\mathrm{cm})$} \\
\hline & & $0-20$ & $20-40$ & $40-60$ & $60-80$ & $80-100$ & $100-120$ & $120-140$ \\
\hline \multirow{2}{*}{ V6 } & 'Hefeng 55 ' & $\begin{array}{c}121.58 \pm \\
4.06 \mathrm{a}\end{array}$ & $\begin{array}{r}13.12 \\
\pm 1.77 \mathrm{a} \\
\end{array}$ & $\begin{array}{c}5.18 \pm \\
0.15 \mathrm{a} \\
\end{array}$ & $\begin{array}{c}3.25 \pm \\
0.75 \mathrm{a} \\
\end{array}$ & $\begin{array}{c}1.46 \pm \\
0.19 \\
\end{array}$ & $\begin{array}{c}0.91 \pm \\
0.50\end{array}$ & 0 \\
\hline & 'Jilv7' & $\begin{array}{c}52.04 \pm \\
1.12 \mathrm{~b}\end{array}$ & $\begin{array}{c}8.98 \pm \\
1.33 \mathrm{a}\end{array}$ & $\begin{array}{c}4.68 \pm \\
1.51 \mathrm{a}\end{array}$ & $\begin{array}{c}1.26 \pm \\
0.72 \mathrm{a}\end{array}$ & 0 & 0 & 0 \\
\hline \multirow{2}{*}{$\mathrm{R} 2$} & 'Hefeng55' & $\begin{array}{r}267.65 \\
\pm 20.36 a \\
\end{array}$ & $\begin{array}{c}31.81 \pm \\
5.95 \mathrm{a} \\
\end{array}$ & $\begin{array}{c}16.14 \pm \\
2.35 \mathrm{a} \\
\end{array}$ & $\begin{array}{r}8.37 \pm \\
0.98 \mathrm{a} \\
\end{array}$ & $\begin{array}{c}4.24 \pm \\
1.02 \mathrm{a} \\
\end{array}$ & $\begin{array}{c}2.26 \pm \\
0.84 \\
\end{array}$ & $\begin{array}{c}1.83 \pm \\
1.22 \\
\end{array}$ \\
\hline & 'Jilv7' & $\begin{array}{c}51.59 \pm \\
1.05 \mathrm{~b} \\
\end{array}$ & $\begin{array}{l}8.14 \pm \\
0.73 b\end{array}$ & $\begin{array}{c}3.33 \pm \\
0.52 \mathrm{a} \\
\end{array}$ & $\begin{array}{c}0.91 \pm \\
0.33 \mathrm{a} \\
\end{array}$ & $\begin{array}{c}0.15 \pm \\
0.08 \mathrm{a} \\
\end{array}$ & 0 & 0 \\
\hline \multirow{2}{*}{$\mathrm{R} 4$} & 'Hefeng55' & $\begin{array}{c}409.74 \pm \\
30.17 \mathrm{a}\end{array}$ & $\begin{array}{c}58.98 \pm \\
6.45 \mathrm{a}\end{array}$ & $\begin{array}{c}26.89 \pm \\
1.91 \mathrm{a} \\
\end{array}$ & $\begin{array}{c}13.03 \pm \\
0.65 \mathrm{a} \\
\end{array}$ & $\begin{array}{c}7.76 \pm \\
0.64 \mathrm{a} \\
\end{array}$ & $\begin{array}{c}6.12 \pm \\
0.56 \mathrm{a} \\
\end{array}$ & $\begin{array}{c}7.18 \pm \\
1.79 \\
\end{array}$ \\
\hline & 'Jilv7' & $\begin{array}{c}55.96 \pm \\
5.08 \mathrm{~b}\end{array}$ & $\begin{array}{c}10.86 \pm \\
1.36 \mathrm{~b}\end{array}$ & $\begin{array}{c}8.86 \pm \\
1.64 \mathrm{a}\end{array}$ & $\begin{array}{c}1.74 \pm \\
0.62 \mathrm{a}\end{array}$ & $\begin{array}{c}0.16 \pm \\
0.03 a\end{array}$ & $\begin{array}{c}0.05 \pm \\
0.01 \mathrm{a}\end{array}$ & 0 \\
\hline \multirow{2}{*}{ R5 } & 'Hefeng55' & $\begin{array}{c}544.01 \pm \\
63.70 \mathrm{a}\end{array}$ & $\begin{array}{c}82.47 \pm \\
13.35 \mathrm{a}\end{array}$ & $\begin{array}{c}28.52 \pm \\
2.59 \mathrm{a}\end{array}$ & $\begin{array}{c}10.08 \pm \\
2.51 \mathrm{a}\end{array}$ & $\begin{array}{r}6.98 \pm \\
1.51 \mathrm{a}\end{array}$ & $\begin{array}{r}9.67 \pm \\
3.19 \mathrm{a}\end{array}$ & $\begin{array}{r}8.95 \pm \\
2.16 \mathrm{a}\end{array}$ \\
\hline & 'Jilv7' & $\begin{array}{c}60.41 \pm \\
3.45 \mathrm{~b}\end{array}$ & $\begin{array}{l}9.11 \pm \\
1.59 \mathrm{~b}\end{array}$ & $\begin{array}{c}6.60 \pm \\
0.13 \mathrm{a}\end{array}$ & $\begin{array}{c}4.56 \pm \\
0.40 \mathrm{a} \\
\end{array}$ & $\begin{array}{c}3.56 \pm \\
0.55 \mathrm{a} \\
\end{array}$ & $\begin{array}{c}3.81 \pm \\
1.59 \mathrm{a} \\
\end{array}$ & $\begin{array}{c}1.27 \pm \\
0.57 \mathrm{a} \\
\end{array}$ \\
\hline \multirow{2}{*}{ R6 } & 'Hefeng55' & $\begin{array}{c}511.85 \pm \\
18.36 \mathrm{a}\end{array}$ & $\begin{array}{c}82.74 \pm \\
5.11 \mathrm{a}\end{array}$ & $\begin{array}{c}28.67 \pm \\
7.34 \mathrm{a}\end{array}$ & $\begin{array}{c}11.75 \pm \\
1.81 \mathrm{a}\end{array}$ & $\begin{array}{c}8.23 \pm \\
2.17 \mathrm{a}\end{array}$ & $\begin{array}{c}5.36 \pm \\
1.08 \mathrm{a}\end{array}$ & $\begin{array}{c}6.78 \pm \\
1.11 \mathrm{a}\end{array}$ \\
\hline & 'Jilv7' & $\begin{array}{c}46.98 \pm \\
2.64 \mathrm{~b}\end{array}$ & $\begin{array}{c}8.92 \pm \\
1.41 \mathrm{~b}\end{array}$ & $\begin{array}{c}5.50 \pm \\
0.60 \mathrm{~b}\end{array}$ & $\begin{array}{c}2.90 \pm \\
1.04 \mathrm{a}\end{array}$ & $\begin{array}{c}2.14 \pm \\
0.71 \mathrm{a}\end{array}$ & $\begin{array}{r}2.36 \pm \\
1.20 \mathrm{a} \\
\end{array}$ & $\begin{array}{c}3.49 \pm \\
1.30 \mathrm{a}\end{array}$ \\
\hline \multirow{2}{*}{ R7 } & 'Hefeng55' & $\begin{array}{c}598.09 \pm \\
32.76 \mathrm{a}\end{array}$ & $\begin{array}{c}87.89 \pm \\
7.38 \mathrm{a}\end{array}$ & $\begin{array}{c}27.42 \pm \\
8.70 \mathrm{a}\end{array}$ & $\begin{array}{c}8.83 \pm \\
1.31 \mathrm{a}\end{array}$ & $\begin{array}{c}5.90 \pm \\
0.34 \mathrm{a}\end{array}$ & $\begin{array}{c}5.81 \pm \\
0.66 \mathrm{a}\end{array}$ & $\begin{array}{c}10.17 \pm \\
2.76 \mathrm{a}\end{array}$ \\
\hline & 'Jilv7' & $\begin{array}{c}40.84 \pm \\
1.72 b\end{array}$ & $\begin{array}{c}8.20 \pm \\
1.13 \mathrm{~b}\end{array}$ & $\begin{array}{c}6.09 \pm \\
0.01 \mathrm{a}\end{array}$ & $\begin{array}{c}1.85 \pm \\
0.91 \mathrm{a}\end{array}$ & $\begin{array}{c}1.17 \pm \\
0.15 \mathrm{a}\end{array}$ & $\begin{array}{c}1.63 \pm \\
0.90 \mathrm{a}\end{array}$ & $\begin{array}{l}1.42 \pm \\
0.34 \mathrm{a}\end{array}$ \\
\hline
\end{tabular}

Data represent average \pm standard error. Distinct letters in the row indicate significant differences. Significant at the 0.05 probability level

\section{Root surface area to root weight ratio}

In horizontal devices, 'Jilv7' had greater root surface area to root weight ratio (AWR) except for R7, and a significantly greater AWR of Jilv7 was found at V6 than 'Hefeng55'. In vertical devices, 'Jilv7' had significantly greater AWR than 'Hefeng55' during all growth stages (Figure 9).

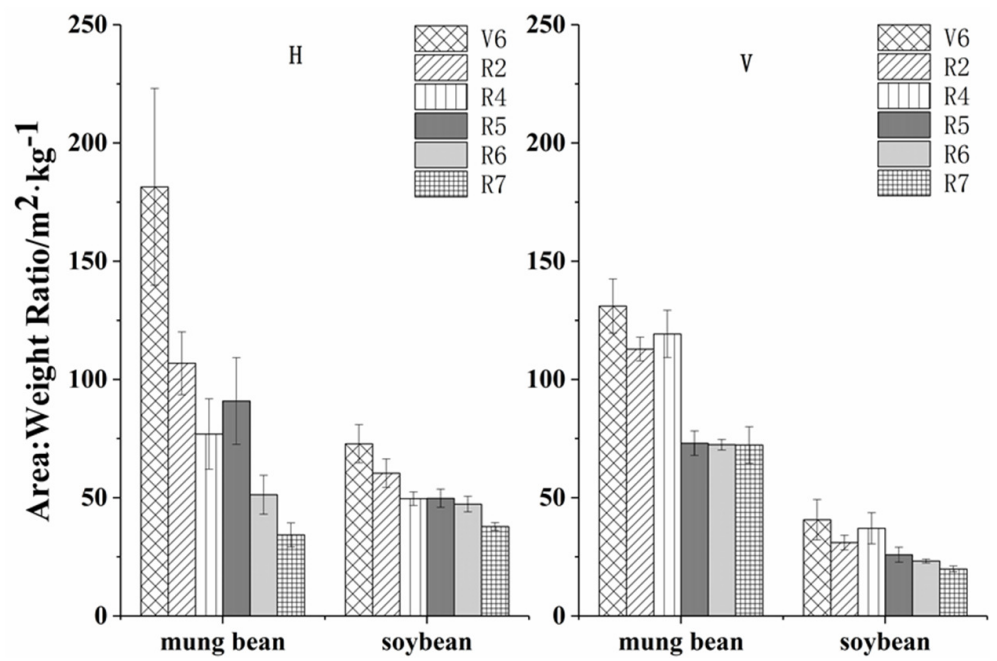

Figure 9. Root surface area to root weight ratio for soybean and mung bean roots in vertical devices and horizontal devices at different growth stages

$\mathrm{V}$ : the vertical device, $\mathrm{H}$ : the horizontal device; Data represent average \pm standard error. Significant at the 0.05 probability level 


\section{Discussion}

In this research, the variation and trend of horizontal distribution of total root length of soybean and mung bean were identical during all growth stages, but soybean had greater total root length than mung bean. In horizontal direction, both soybean and mung bean had the largest proportion of root length in $15-20 \mathrm{~cm}$ soil layer followed by $10-15,20-25$ and $5-10 \mathrm{~cm}$ soil layers, and the minimum proportion of root length was found in $0-5 \mathrm{~cm}$ soil layer. Root length of soybean in vertical $0-20 \mathrm{~cm}$ and $20-40 \mathrm{~cm}$ soil layers reached $56.8 \%$ and $23.2 \%$ of the total root length, respectively, which were higher than $51.2 \%$ and $22.9 \%$ of mung bean in the same soil layer. This is similar to the finding of Gao et al. (2010). However, both the ratio of root length to total root length of mung bean in vertical $40-60$ and $60-80 \mathrm{~cm}$ soil were greater than those of soybean. In soil layers below $80 \mathrm{~cm}$ in vertical direction, the root length percentages of soybean and mung bean were similar. The root length of soybean and mung bean were mainly concentrated in $0-40 \mathrm{~cm}$ vertical soil layer, and root length of mung bean was more evenly distributed in the vertical direction compared with soybean.

The root length density of the crop could be used to reflect extension and distribution of crop root (Adiku et al., 2001; Zhu, 2010; Liu et al., 2011). With the deepening of the soil layer, root length density of soybean and mung bean decreased gradually. One possible explanation for this phenomenon is that mechanical impedance limited the extension of the root system (Logsdon et al., 1987).

The distribution of crop root volume is critical to growth, development and yield formation of crop (Rao and Ito, 1998). The trend of total root volume of mung bean was more stable than that of soybean, but the total root volume of soybean was greater than that of mung bean during all growth stages. Spatial distribution of root showed that the root volume of mung bean was more concentrated in $0-5 \mathrm{~cm}$ horizontal soil layer compared with soybean, but soybean root volume tended to develop laterally. Both soybean (26.6\%) and mung bean (23.9\%) had the largest proportion of root volume in horizontal $15-20 \mathrm{~cm}$ soil layer. The ratio of root volume to total root volume in horizontal $0-5 \mathrm{~cm}$ soil layer of mung bean was greater than that of soybean, but the proportion of soybean in other horizontal soil layers were greater than those of mung bean. In vertical direction, soybean and mung bean were similar in root volume percentage in $0-20 \mathrm{~cm}$ vertical soil layer, and both of them had the greatest proportion in this layer. This is similar to the finding of Gan (2011). The root volume percentage of soybean in vertical $20-40 \mathrm{~cm}$ soil layer was higher than that of mung bean, but in the subsequent $40-80 \mathrm{~cm}$ vertical soil layer, mung bean had a larger root volume percentage compared with soybean. This showed that the difference of root volume distribution between soybean and mung bean was mainly concentrated in the upper middle soil layers in vertical direction. Compared with mung bean, soybean had a larger proportion of root volume in the upper soil layer, while proportion of mung bean root volume was higher than that of soybean in the middle soil layer.

In horizontal spatial distribution, the root surface area of soybean and mung bean were mainly distributed in the soil layer of 10-25 cm. Compared with soybean, mung bean had a larger percentage of root surface area in horizontal $0-10 \mathrm{~cm}$ soil layer. In vertical direction, soybean and mung bean had a similar distribution of root surface area in the upper, middle and lower layers. For root dry weight distribution, the dry root weight of soybean (49.8\%) and mung bean $(57.8 \%)$ were mainly distributed in $0-5 \mathrm{~cm}$ horizontal soil layer, and both dry weight of soybean and mung bean in vertical $0-20 \mathrm{~cm}$ soil layer reached more than $70 \%$ of the total dry weight, more than $82 \%$ of total root dry weight in vertical $0-40 \mathrm{~cm}$ soil layer. This is similar to the finding of Mitchell and Russell (1971) who found the highest proportion of root dry weight density in vertical $0.23 \mathrm{~m}$ soil layer.

A low AWR indicates either a thicker root system or roots with higher specific density (Benjamin and Nielsen, 2006). Mung bean had greater AWR than soybean during all growth stages. This showed mung bean had a finer root system or roots with lower specific density. From V6 to R7, soybean and mung bean AWR decreased indicating a thickening or densification of the root material.

In horizontal devices, we found that the maximum of total root length, total root surface area, and total root volume of soybean and mung bean were at R5. However, in vertical device, the maximum of total root 
length, total root surface area, and total root volume of soybean were at R5. But for mung bean, the maximum of total root length and total root volume were found at R4 and the maximum total root surface was at $\mathrm{R} 2$. The reason may be that limiting the extension of roots in horizontal direction accelerated the aging process of mung bean roots, but soybean roots showed stronger adaptability than mung bean.

\section{Conclusions}

Compared with mung bean, soybean had a much larger root system during development; In horizontal direction, root system was mainly concentrated in the $0-5 \mathrm{~cm}$ soil layer, but soybean root tended to be more laterally developed compared with mung bean. In vertical direction, the distribution of mung bean root was more uniform than that of soybean; With a greater AWR, mung bean had a finer root system than soybean.

Authors' Contribution

HZ: Collection of samples, data collection and analysis, article writing; DZ and NF: Guidance on methods. All authors read and approved the final manuscript.

\section{Acknowledgements}

The authors gratefully acknowledge the language modification by Seth Kutikoff from Kansas State University, and financial support provided by the project of Heilongjiang Province Outstanding Youth Science Foundation (JC201309).

\section{Conflict of Interests}

The authors declare that there are no conflicts of interest related to this article.

\section{References}

Adiku SG, Ozier-Lafontain H, Bajazet T (2001). Patterns of root growth and water uptake of a maize-cowpea mixture grown under greenhouse conditions. Plant and Soil 235:85-94. https://doi.org/10.1023/A:1011847214706

Atta BM, Mahmood T, Trethowan TM (2013). Relationship between root morphology and grain yield of wheat in northwestern NSW, Australia. Australian Journal of Crop Science 7:2108-2115.

Benjamin J, Nielsen DC (2006). Water deficit effects on root distribution of soybean, field pea and chickpea. Field Crop Research 97:248-253. https:doi.org/10.1016/j.fcr.2005.10.005

Calonego JC, Rosolem CA (2010). Soybean root growth and yield in rotation with cover crops under chiseling and notill. European Journal of Agronomy 33:242-249. https://doi.org/10.1016/j.eja.2010.06.002

Ehdaie B, Merhaut DJ, Ahmadian S, Hoops AC, Khuong T, Layne AP, Waines JG (2010). Root system size influences water-nutrient uptake and nitrate leaching potential in wheat. Journal of Agronomy and Crop Science 196:455466. https://doi.org/10.1111/j.1439-037X.2010.00433.x

Eissenstat DM, Yanai RD (2002). Root life span, efficiency, and turnover. In Plant Roots, the Hidden Half. CRC Press, New York pp 221-238.

Fan J, McConkey B, Wang H, Janzen H (2016). Root distribution by depth for temperate agricultural crops. Field Crops Research 189:68-74. https://doi.org/10.1016/j.fcr.2016.02.013

Fang S, Gao X, Deng Y, Chen X, Liao H (2011). Crop root behaviour coordinates phosphorus status and neighbours: from field studies to three-dimensional in situ reconstruction of root system architecture. Plant Physiology 155:1277-1285. https://doi.org/10.1104/pp.110.167304 
Farmaha BS, Fernández FG, Nafziger ED (2012). Distribution of soybean roots, soil water, phosphorus and potassium concentrations with broadcast and subsurface-band fertilization. Soil Science Society of America Journal 76:1079-1089.

Gan Y, Campbell CA, Liu L, Basnyat P, Mcdonald CL (2009). Water use and distribution profile under pulse and oilseed crops in semiarid northern high latitude areas. Agricultural Water Management 96:337-348. https://doi.org/10.1016/j.agwat.2008.08.012

Gan Y, Liu L, Cutforth H, Wang X, Ford G (2011). Vertical distribution profiles and temporal growth patterns of roots in selected oilseeds, pulses and spring wheat. Crop and Pasture Science 62:457-466.

Gao Y, Duan A, Qiu X, Liu Z, Sun J, Zhang J, Wang H (2010). Distribution of roots and root length density in a maize/soybean strip intercropping system. Agricultural Water Management 98:199-212. https://doi.org/10.1016/j.agwat.2010.08.021

Liu L, Gan Y, Bueckert R, Van RK (2011). Rooting systems of oilseed and pulse crops. ii: vertical distribution patterns across the soil profile. Field Crops Research 122:248-255. https://doi.org/10.1016/j.fcr.2011.04.003

Logsdon SD, Reneau RB, Parker JC (1987). Corn seedling root growth as influenced by soil physical properties. Agronomy Journal 79:221-224. https://doi.org/10.2134/agronj1987.00021962007900020010x

Lynch J (1995). Root architecture and plant productivity. Plant Physiology 109:7-13.

Mitchell RL, Russell WJ (1971). Root development and rooting patterns of soybean (Glycine max (L.) Merrill) evaluated under field conditions. Agronomy 63:313-316. https://doi.org/10.2134/agronj1971.00021962006300020034x

Pedersen P, Kumudini S, Board J, Conley S (2004). Soybean growth and development. University Extension. Iowa State University.

Rao TP, Ito O (1998). Differences in root system morphology and root respiration in relation to nitrogen uptake among six crop species. Japan Agricultural Research Quarterly 32:97-103.

Vamerali T, Saccomani M, Bona S, Mosca G, Guarise M, Ganis A (2003). A comparison of root characteristics in relation to nutrient and water stress in two maize hybrids. Plant and Soil 255:157-167. https://doi.org/10.1023/A:1026123129575

Waisel Y, Eshel A (2002). Functional diversity of various constituents of a single root system. In: Plant Roots CRC Press, New York pp 157-174.

Zhu J, Brown KM, Lynch JP (2010). Root cortical aerenchyma improves the drought tolerance of maize (Zea mays L.). Plant, Cell and Environment 33:740-749. https://doi.org/10.1111/j.1365-3040.2009.02099.x

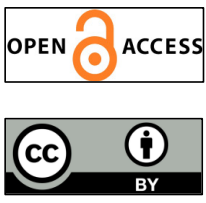

The journal offers free, immediate, and unrestricted access to peer-reviewed research and scholarly work. Users are allowed to read, download, copy, distribute, print, search, or link to the full texts of the articles, or use them for any other lawful purpose, without asking prior permission from the publisher or the author.

License - Articles published in Notulae Botanicae Horti Agrobotanici Cluj-Napoca are Open-Access, distributed under the terms and conditions of the Creative Commons Attribution (CC BY 4.0) License.

(c) Articles by the authors; UASVM, Cluj-Napoca, Romania. The journal allows the author(s) to hold the copyright/to retain publishing rights without restriction. 